\title{
The Peculiar Globular Cluster System of the So Galaxy NGC 7457
}

\author{
Laura Chomiuk $^{1}$, Jay Strader ${ }^{2,3}$, \& Jean P. Brodie ${ }^{4}$ \\ chomiuk@astro.wisc.edu, jstrader@cfa.harvard.edu, brodie@ucolick.org
}

\begin{abstract}
We present $H S T$ photometry and Keck spectroscopy of globular clusters (GCs) in the nearby S0 galaxy NGC 7457. The $V-I$ color-magnitude diagram of GCs lacks the clear bimodality present in most early-type galaxies; there may be a significant population of intermediate-color objects. Of 13 spectroscopically-observed GCs, two are unusually metal-rich and feature bright [O III] emission lines. We conclude that one probably hosts a planetary nebula and the other a supernova remnant. Such emission line objects should be more common in an intermediate-age stellar population than in an old one. We therefore suggest that, in addition to the typical old metal-rich and old metal-poor GC subpopulations, there may be a third subpopulation of intermediate age. Such a subpopulation may have been formed $\sim 2-3$ Gyr ago, in the same star-forming event that dominates the stellar population of the center of the galaxy.
\end{abstract}

Subject headings: Galaxies: Star Clusters, Galaxies: Individual: NGC Number: NGC 7457, Galaxies: Interactions, Supernova Remnants, Planetary Nebulae

\section{Introduction}

Extragalactic globular clusters (GCs) provide a unique window onto the stellar populations of nearby galaxies, as they trace and simplify star formation episodes in nearly all galaxies from dwarfs to giant ellipticals. If a subpopulation of GCs is found to have a given age and metallicity, one can extrapolate that there is a significant component of the parent galaxy's stellar population with the same characteristics (Brodie \& Strader 2006, Harris 2001, and references therein). In this way, GCs are valuable fossils which allow us to dissect the complex star formation histories of galaxies in our local universe.

S0 galaxies stand at a junction in the Hubble tuning fork, and they may symbolize a transition in the dominant processes of galaxy evolution. As summarized by Kormendy \& Kennicutt (2004),

\footnotetext{
${ }^{1}$ University of Wisconsin-Madison, Madison, WI 53706

${ }^{2}$ Harvard-Smithsonian Center for Astrophysics, Cambridge, MA 02138

${ }^{3}$ Hubble Fellow

${ }^{4}$ UCO/Lick Observatory, University of California, Santa Cruz, CA 95064
} 
in the past, galaxy evolution was dominated by the violent processes of hierarchical merging. In the future, the main processes affecting galaxies will be more gradual, involving the detailed internal dynamics of galaxies themselves. Today, the relative importance of secular and merging processes appears to be a function of Hubble type, as the bulges of late-type spirals tend to be formed by gas and stars funneled along disk asymmetries like bars, while ellipticals are thought to have been formed by mergers and violent relaxation. However, the relative importance of these mechanisms is still unclear in early-type spirals and lenticulars. The bulges of these galaxies appear to be mostly "classical" (formed by mergers), but secular evolution certainly plays a role in many systems.

The SA0 galaxy NGC 7457, at first glance, looks like a perfect example of a normal lenticular, with a classical de Vaucouleurs bulge and an exponential disk (Kormendy 1977). Basic data for this galaxy are listed in Table 1. No dust and very little cold gas have been detected in NGC 7457 (Peletier et al. 1999, Welch \& Sage 2003, Sage \& Welch 2006). It is one of the nearest S0 galaxies, at a distance of $13 \mathrm{Mpc}$ (Cappellari et al. 2006). However, on closer inspection, NGC 7457 has shown several peculiarities in its dynamics and stellar populations, which have made it the center of much debate. Kormendy \& Illingworth (1983) observed that the bulge of NGC 7457 has an unusually low velocity dispersion for its luminosity. This suggested that the bulge has the more ordered dynamics of a disk, and is predominantly supported by rotation. However, Dressler \& Sandage (1983) noticed that the bulge also appeared to have unusually low circular velocities. This puzzling contradiction has been explained by SAURON data, which show that NGC 7457 actually has a counter-rotating core (Sil'chenko et al. 2002, Emsellem et al. 2004). A counter-rotating core is a possible consequence of a merger. Additionally, the core has a distinct stellar population from the bulge. Sil'chenko et al. (2002) found that the bulge has an intermediate luminosity-weighted age of 5-7 Gyr, while the compact nucleus (radius of $\left.1.5^{\prime \prime}\right)$ contains a younger (2-2.5 Gyr), chemically distinct stellar population.

GCs may be an interesting diagnostic of the relative significance to star formation of violent vs. secular processes. If stars in a galaxy were formed from a gaseous merger, stars will have formed in a burst, and GCs along with them. If stars form more quiescently, then GCs may not accompany them (Brodie \& Strader 2006). Therefore, one of the goals of this study is to search for GCs of intermediate age to place constraints on the bulge and nucleus formation in NGC 7457.

Several claims of unusual GC phenomena have been made for the NGC 7457 system by Chapelon et al. (1999). They obtained ground-based B, V, and I photometry, but did not find the usual color bimodality in the GC population. This color bimodality is observed in the Milky Way and many other galaxies of all morphological types, and generally represents two old stellar populations with different metallicities (see Brodie \& Strader 2006, and references therein). In the Milky Way, these populations have $[\mathrm{Fe} / \mathrm{H}]=-1.5$ (for the blue peak), and $[\mathrm{Fe} / \mathrm{H}]=-0.5$ (for the red peak). Chapelon et al. found a broad unimodal distribution centered at colors corresponding to $[\mathrm{Fe} / \mathrm{H}]=-1.0$, with a width of $\sigma([\mathrm{Fe} / \mathrm{H}])=0.6$ dex. The authors claim that this width is too narrow to be composed of two unresolved GC populations, and that the mean color is too red to accommodate a significant population of blue (metal-poor) GCs. Provocative as these results are, the 
ground-based data have a small sample size and large photometric errors. A solid understanding of the NGC 7457 GC system requires a larger sample, more accurate photometry, and spectroscopy.

In Section 2, we discuss archival Hubble Space Telescope (HST) photometry of the NGC 7457 GC system. In Section 3 is presented Keck/LRIS spectroscopy for 13 GCs. Section 4 provides an analysis of the radial velocities obtained from the spectroscopy, while in Section 5 we present our techniques for constraining the spectroscopic GCs' stellar populations. In Sections 6 and 7 we discuss the properties of the stellar populations. In Section 8, we discuss two curious GCs with emission lines. Finally, in Section 9, we synthesize our results and draw conclusions.

\section{HST Photometry}

\subsection{WFPC2 Data}

We downloaded archival HST/WFPC2 images of the center of NGC 7457 taken in Cycle 4. These images have total exposure times of $1250 \mathrm{sec}$ in $\mathrm{F} 814 \mathrm{~W}$ and $1330 \mathrm{sec}$ in $\mathrm{F} 555 \mathrm{~W}$, and were pipeline-processed in the usual way before retrieval. The individual images were registered and coadded to reject cosmic rays. Detection of GC candidates was carried out on $20 \times 20$ pixel median-subtracted images. Because of the low density of GC candidates, aperture photometry is sufficient. We measured magnitudes through apertures with 3 pixel radii (corresponding to $0.3^{\prime \prime}$ for the WF chips and $0.14^{\prime \prime}$ for the PC chip). These were corrected to standard $V$ and $I$ magnitudes using the updated equations of A. Dolphin 1 (see Dolphin 2000), except that no correction for charge transfer efficiency was made due to the galaxy background.

Corrections from 3 to 5 pixel apertures for the WF chips were taken from Larsen \& Brodie (2000), and assume GC effective radii of 3 pc and a galaxy distance of $10 \mathrm{Mpc}$. These are -0.14 mag in $V$ and -0.166 in $I$. As noted by Larsen \& Brodie, these corrections will systematically underestimate the magnitudes of very large GCs, but the colors are unaffected. An additional -0.1 mag was added to correct the magnitudes to a nominal infinite aperture (Holtzman et al. 1995). Larsen \& Brodie (2000) did not calculate aperture corrections for the PC chip, so we used the equations in Kundu \& Whitmore (2001), which give corrections of -0.44 mag in $V$ and -0.49 mag in $I$. These are from 3 pixels to infinity (thus including the Holtzman et al. correction) and assume a galaxy distance of $13 \mathrm{Mpc}$. Finally, the $V$ and $I$ magnitudes were corrected for extinction, using values of $A_{V}=0.172 \mathrm{mag}$ and $A_{I}=0.101 \mathrm{mag}$, from the maps of Schlegel et al. (1998). All plotted and tabulated magnitudes are extinction-corrected.

GC candidate sizes were estimated using the ishape algorithm (Larsen 1999), which convolves subsampled TinyTim model PSFs with King models of varying effective radii to find the best fit to the data. We used a fixed model concentration $c=r_{\text {tidal }} / r_{\text {core }}=30$. The resulting initial list of

\footnotetext{
${ }^{1}$ http://purcell.as.arizona.edu/wfpc2_calib/
} 
GC candidates was trimmed using a color cut of $0.7<V-I<1.5$ and an upper size limit of 25 pc.

These criteria left us with a final list of $75 \mathrm{GC}$ candidates. The associated $V$ vs. $V-I$ color-magnitude diagram is shown in Figure 1, and Figure 2 shows a histogram of the $V-I$ color distribution. It is difficult to draw detailed conclusions from these plots due to the small number of objects and the moderately shallow nature of the imaging. Nonetheless, it is clear that there is a broad distribution of GC colors, with perhaps two distinct peaks: a dominant population of GCs around $V-I \sim 1.0$ and a smaller, redder set of objects. A clearly bimodal distribution of GCs, like that seen in most early-type galaxies (Brodie \& Strader 2006), is not obvious. We used the program Nmix (Richardson \& Green 1997; also see discussion in Strader et al. 2006) to perform mixture modeling on the colors of GCs with $V<23.5$; this analysis yields a strong preference for a unimodal distribution peaked at $V-I=1.02$.

These results are generally consistent with those of Chapelon et al. (1999), who used groundbased imaging of NGC 7457 to claim a broad GC color distribution centered around $V-I=1.04$. They suggested the existence of two subpopulations of GCs, but with a smaller peak separation than is typical for massive early-type galaxies.

One plausible alternative scenario is that there is an underlying bimodal distribution of old metal-poor and metal-rich GCs (at the typical metallicities) with an added younger component at intermediate colors. According to the models of Maraston (2005), a single stellar population (SSP) with solar metallicity, an age of $1.5 \mathrm{Gyr}$, and a Kroupa initial mass function should yield a color of $V-I=1.02$; at lower metallicities, this color corresponds to somewhat older ages. Such an age would be consistent with the spectroscopic age of the center of the galaxy (Sil'chenko et al. 2002; see Section 1). However, if this younger component formed with a standard GC mass function, the clusters ought to extend to brighter magnitudes than the old GCs (as seen, for example, in the merger remnant NGC 1316; Goudfrooij et al. 2001). This behavior is not obvious in Figure 1, perhaps indicating that there are only a small number of intermediate-age GCs, or that they did not form with the GC mass function typical in luminous galaxies. Small number statistics could also play a role.

Even if there is a significant subpopulation of intermediate-age GCs, both of the old GC subpopulations are probably still present in NGC 7457. It is unlikely that all of the red GCs are of intermediate or younger age, given that they extend to rather red colors $(V-I>1.2)$ difficult to produce except at old ages. In addition, no luminous galaxy has been convincingly shown to lack metal-poor GCs. If we model the GC colors with the restriction that there are three subpopulations, the color peaks lie at $V-I=0.92,1.03$, and 1.16 (with relative contributions of $33 \%, 41 \%$, and $25 \%$ ). The extreme red and blue peaks are consistent within $\sim 0.02$ mag of the GC color-galaxy luminosity relations for the old metal-rich and metal-poor subpopulations (Strader et al. 2004). We thus consider a three-component GC system to be the most likely explanation, though by no means certain. Given the limited spatial coverage of the WFPC2 data, the small 
number of objects, and the uncertain mass function of the hypothesized intermediate-age GCs, the percentages above should be considered very preliminary, but they do indicate that the younger objects might represent a substantial fraction of the overall GC system.

\subsection{ACS/HRC Data}

As this manuscript was nearing submission, ACS/HRC UV imaging of the central regions of NGC 7457 became publicly available. This imaging consisted of 2552 sec in F250W, 1912 sec in F330W, and $432 \mathrm{sec}$ in F555W. Since this imaging partially overlaps the older WFPC2 imaging discussed above, we decided to analyze it to increase our wavelength baseline, potentially increasing our ability to detect intermediate-age GCs. While the F555W image is useful for morphological classification of GC candidates, it is not substantially deeper than the WFPC2 image and so we did not perform photometry on it.

The analysis process was similar to that used for the WFPC2 images. We searched for GC candidates on median-subtracted, pipeline-processed images. These catalogs were cross-matched with the WFPC2 candidate list. For the F250W image there were only two matches, and both objects appeared to be very faint, so we do not discuss the F250W data further. However, there were eleven clear detections on the F330W image. Two of these were very close to each other and to the center of the galaxy; the high background makes accurate photometry impossible. They are located at a projected distance of $1.4^{\prime \prime}(\sim 88 \mathrm{pc})$ from the galaxy center, but are separated from each other by only $\sim 0.2^{\prime \prime}(13 \mathrm{pc})$. Assuming these are true GCs, it is very unlikely that they form a bound pair; the merging timescale would be only a few $\times 10^{7} \mathrm{yr}$ (Minniti et al. 2004; Sugimoto \& Makino 1989).

For the other nine candidates, we performed aperture photometry in F330W with a 5-pixel aperture; this is a compromise between the small pixel scale of the HRC (compared to WPFC2 and the intrinsic cluster sizes of $\sim 0.03-0.05^{\prime \prime}$ ) and the larger instrumental PSF in the ultraviolet. Since the GCs are resolved, the "standard" encircled energy values cannot be used for aperture corrections; instead, these were estimated by convolving a GC of typical size (2.5 pc at $13 \mathrm{Mpc}$ ) with a TinyTim ACS/HRC PSF using ishape (Larsen 1999). The correction from our 5 pixel aperture to one of $0.5^{\prime \prime}$ is $-0.257 \mathrm{mag}$. We then used the Sirianni et al. (2005) value of -0.117 mag to correct from a $0.5^{\prime \prime}$ to a nominally infinite aperture. The final correction is for foreground reddening; using the Schlegel et al. (1998) value of $E(B-V)=0.052$ and the conversion factor in Sirianni et al. $(2005)$ yields $A_{F 330}=0.26$. The extinction and aperture corrections total -0.634 .

The dereddened $F 330 W-V$ colors of these nine candidate GCs are given in Table 2. In Figure 3 we show a $F 330 W-V$ vs. $V-I$ color-color plot of the clusters. Overplotted are model isochrones from Girardi et al. (2002) for two ages: 2 Gyr and $11 \mathrm{Gyr}$, with metallicities ranging from $[Z / \mathrm{H}]=$ -1.7 to solar. The two objects that are spectroscopically-confirmed members are marked separately. Seven of the objects are consistent with the model isochrones, with four of the seven consistent 
with either old ages and low metallicities or with younger ages (none of these four have spectra). The other three objects are too red in both $F 330 W-V$ and $V-I$ to be of intermediate age. The single object marked with a circle (ID L1 in Table 2) is the most luminous in the sample, with $V=20.5$, and is also unusually large, with an effective radius of $\sim 20 \mathrm{pc}$. Its UV-optical colors are marginally more consistent with an intermediate age than the other candidates. The luminosity and size of the cluster are reminiscent of NGC 2419 in the Milky Way, which has been argued to be the stripped core of a dwarf galaxy (van den Bergh \& Mackey 2004). Spectroscopy of this object would be desirable to confirm membership in NGC 7457 and to assess its age and metallicity.

Two candidates do not fall on the isochrones. One is the confirmed cluster GC11. Morphologically this object is quite odd; it is notably asymmetric, with a "tail"-like structure to the NE, and would likely be discarded as a background galaxy in a typical study. Given its unusual colors and morphology, we suggest that this is a true GC superimposed on a background object. The other object that is not on the isochrones (UV4) looks compact and symmetric in all of the ACS/HRC images. It is probably a compact background galaxy, though we cannot exclude an unidentified error with the photometry.

\section{Spectroscopic Observations and Data Reduction}

We observed the NGC 7457 GC system with Keck I and the Low-Resolution Imaging Spectrometer (LRIS; Oke et al. 1995) in multi-slit mode on October 21-22, 2003. A total of 15 exposures were taken, each of 1800 seconds duration, for an equivalent exposure time of 450 minutes. We also obtained long-slit spectra of one flux standard star, BD+28 4211 from Massey \& Gronwall (1990), and four Lick/IDS standard stars, HR 2153, HR 2459, HR 7429, and HR 8165 (Worthey et al. 1994). All data were obtained through a 600 line $\mathrm{mm}^{-1}$ grism blazed at $4000 \AA$ on the blue side, and an 831 line $\mathrm{mm}^{-1}$ grism blazed at $8200 \AA$ on the red side, with a dichroic splitting the incoming light at $6800 \AA$. Slits were $0.8^{\prime \prime}$ in width. On the blue side, this provides a reciprocal dispersion of $0.63 \AA$ pixel $^{-1}$ and a spectral resolution of $2.8 \AA$. The wavelength range of a slit in the center of the mask is 3300-5880 $\AA$, but this range can vary by several hundred angstroms depending on the positions of individual slitlets on the mask. The red side of our spectra covers the wavelength range 7100-9100 $\AA$ on average, in order to include the Ca II triplet. Unfortunately, the fringing proved too severe for a proper reduction of the red spectra; here we present only the blue spectra.

Our data were reduced using the standard image and spectral reduction tools in IRAF. The raw images were bias-subtracted and flat-fielded using a normalized dome flat. The intensity of the quartz lamp varied strongly with wavelength, so the large-scale spectral response was removed from the dome flats before they were applied. Also, the quartz lamp provided very little signal in the far blue, so we were not able to flat-field at all at the shortest wavelengths.

We then extracted our spectra using apall. We wavelength-calibrated the spectra using HgNeArCdZn comparison arc lamps, and applied small zero-point corrections of a few angstroms by 
aligning the bright O I skyline to its proper wavelength of $5577.34 \AA$. At this point, we combined the 15 spectra from the separate exposures by averaging them together with sigma-clipping. The quality of these spectra are estimated in Table 3 , where the signal-to-noise ratio (S/N) per $\AA$ around $\mathrm{H} \beta$ is listed for the spectra. We flux-calibrated our averaged spectra using the flux standard $\mathrm{BD}+28^{\circ}$ 4211.

We chose GCs for spectroscopy primarily from the WFPC2 imaging described in Section 2.1. Given the suggestion of Sil'chenko et al. (2002) that NGC 7457 may be an intermediate-age merger remnant, one of our primary goals was the identification of intermediate-age GC candidates. Thus red GCs were preferentially selected for inclusion in the slit-mask, though a number of blue objects were included as well to fill in the mask. Slits were placed on 17 WFPC2 objects. From these, we obtained good spectra for 13 GCs and the nucleus of NGC 7457 itself. The other three slits had various issues: one contained a GC, but was so near the nucleus of NGC 7457 that the background was very high, and it proved impossible to subtract the galaxy's spectrum properly. Another slit also contained a GC, but the data were vignetted by a bracket in the spectrograph. Finally, one of the slits contained an object with a velocity of $-412 \mathrm{~km} \mathrm{~s}^{-1}$, most likely a Galactic star. Additional GC candidates in the outer parts of the galaxy were selected from ground-based imaging; unfortunately, our spectroscopy indicates that all of these extra candidates are contaminants ( 7 stars and 2 background galaxies).

Basic information about the 13 GCs in our sample can be found in Table 3 . The radial velocities and $\mathrm{S} / \mathrm{N}$ values were measured on our Keck LRIS spectra. Figure 4 shows two such spectra, for a prototypical metal-poor GC, namely GC9, and a metal-rich GC, GC5. The reader should also be aware of two interesting outliers, GC6 and GC7, which not only exhibit the usual absorption-line spectra, but also [O III] $\lambda \lambda 4959,5007$ emission lines. The spectra for these two GCs can be seen in Figure 5 (along with GC5 for comparison), and will be discussed in Section 8.

\section{Kinematics}

Radial velocities for our 13 GCs and the center of NGC 7457 are listed in Table 3. Using fxcor in IRAF, we cross-correlated our spectra with MILES SSP models (Sánchez-Blázquez et al. 2006; A. Vazdekis et al. 2008, in preparation) to determine radial velocities and errors. We bootstrapped to

estimate global kinematic quantities. We find a mean radial velocity of $856 \pm 20 \mathrm{~km} \mathrm{~s}^{-1}$. Given the rotation of the GC system (see below), and the asymmetric spatial distribution of our GC sample, this value is consistent with our measured radial velocity of the center of NGC $7457,789 \pm 15 \mathrm{~km}$ $\mathrm{s}^{-1}$. The velocity dispersion of the 13 GCs is $69 \pm 12 \mathrm{~km} \mathrm{~s}^{-1}$.

Figure 6 shows the spatial distribution of the GCs and their radial velocities superimposed on a representative isophote of the galaxy. The general impression is that the GC system is rotating around the minor axis of the galaxy. Fitting a simple solid-body rotation curve to the weighted velocities gives a best-fit axis of $\sim 160^{\circ}$, generally consistent with the photometric position angle of 
$\sim 130^{\circ}$ for the galaxy itself. The GCs are also rotating in the same sense (with the southern objects blueshifted) as the galaxy (Emsellem et al. 2004). Of course, if there is a substantial degree of rotation in the GC system, the velocity dispersion quoted above should be taken only as an upper limit to the pressure support for the kinematics of the GCs.

Given the small number of GCs and their asymmetric distribution, we see little value in detailed analysis of the kinematics. The system appears more rotationally supported than most early-type galaxies - the notable exception being the Virgo dwarf elliptical VCC 1087 (Beasley et al. 2006). NGC 7457 may provide additional evidence that disky populations of GCs are common in lower-mass galaxies.

\section{Techniques for Fitting SSP Models to GC Spectra}

\subsection{Lick/IDS Index Measurements}

The Lick/IDS system of absorption-line indices provides a standardized framework in which one can derive metallicities, ages, and abundance ratios for stellar populations. The Lick/IDS system was originally created by Burstein et al. (1984), and has been continuously revised through the years (Worthey et al. 1994; Trager et al. 1998). Considerable attention has also been paid to modeling the Lick/IDS indices and their variations with stellar age and metallicity (Bruzual \& Charlot 2003; Maraston \& Thomas 2000; Maraston, Greggio, \& Thomas 2001; Worthey et al. 1994; Fritze-v. Alvensleben \& Burkert 1995). Lick/IDS indices have been very successful at lifting the age-metallicity degeneracy, and can distinguish, for example, intermediate-age (3-4 Gyr) metal-rich GCs from their much older counterparts, which would be indistinguishable from optical photometry alone (Strader et al. 2003b).

In order to match our spectra to the standardized Lick/IDS system, we smoothed our fluxcalibrated spectra with a wavelength-dependent Gaussian kernel as prescribed in Worthey \& Ottaviani (1997) and for the reasons described in Larsen \& Brodie (2002) and Strader et al. (2003b). All indices and errors were measured using the $\mathrm{C}++$ program Indexf (Cardiel et al. 1998). We measured indices on $13 \mathrm{GCs}$ and on the inner $\sim 1.25^{\prime \prime}$ of the galaxy itself, for comparison. We also measured indices for four Lick standard stars, and calculated mean offsets between our measurements and the Lick system. These small shifts were applied to the Lick indices of our 14 objects, listed in Table 4. Additionally, for each index, we determined the error in these offset by calculating the standard deviation of the difference between our measurements and the standard Lick values. These standard deviations were added in quadrature to the Indexf errors to give the errors listed in Table 4. 


\subsection{Line Diagnostic Plots}

Plotting Lick indices against one another allows us to visualize the distributions of GC ages, metallicities, and abundance ratios. Such plots can be seen as Figures 7-10. Often, individual indices are combined to form a more robust composite index-for example, $<\mathrm{Fe}>$ (the average of Fe5270 and Fe5335). Model isochrone and isometallicity lines are overplotted. In this study, we use the SSP models of Thomas et al. (2003) and Thomas et al. (2004).

Although Mgb is commonly used as the index sensitive to $\alpha$ elements, in this study we will use Mg2, as it appears that unknown systematics are affecting our Mgb measurements. Figure 7a plots the Mg2 index against the Mgb index, and at low metallicities it is clear that the Mgb index sits below the model predictions. GCs from published data sets (NGC 4365 (Brodie et al. 2005); Fornax (Strader et al. 2003a), NGC 1407 (Cenarro et al. 2007)) sit comfortably along this line, thus implying our data are faulty at Mgb. There is a sky line at $\lambda 5202 \AA$ in the $\mathrm{Mg} b$ and $\mathrm{Mg} 2$ bandpasses; we tried masking this line and remeasuring the spectra, and the index values changed very little. When we compare Mg2 with Mg1 in Figure 7b, the measurements agree well with model predictions. When Mg2 is compared with other $\alpha$-element indicators, like CN2 and Ca 4227, it remains consistent with the models. Thus, here and throughout this paper, we will make use of the Mg2 index while rejecting the $\mathrm{Mg} b$ measurements.

\subsection{Composite Metallicities}

We derived metallicity estimates for the individual NGC 7457 GCs using the composite technique described in Strader et al. (2007). Briefly, Galactic GCs are used to define second-order polynomial relations between the strength of individual Lick indices and total metallicity. The estimates from the different indices are then combined to yield a robust measure of the GC metallicity with an associated error. In this study, the Balmer lines were excluded from our calculations due to emission-line contamination in two objects.

Table 3 lists the metallicity estimates for the 13 GCs and the center of NGC 7457 itself. These composite metallicities are calibrated for old stellar populations with metallicities roughly within the range $-1.8 \lesssim[\mathrm{m} / \mathrm{H}] \lesssim 0$; there may be systematic offsets for younger ages and very metal-rich or metal-poor objects.

\subsection{Multi-index Fits via $\chi^{2}$ Minimization}

We used an additional route to take advantage of the information provided by many indices - a $\chi^{2}$ minimization algorithm described by Proctor et al. (2004), which simultaneously finds bestfitting GC ages, $[\mathrm{Z} / \mathrm{Fe}]$, and $[\alpha / \mathrm{Fe}]$. This algorithm uses a grid of models from Thomas et al. (2003, 2004) to fit between 8 and 15 Lick indices. 
The results of this analysis are presented in Table 5. Note that the multi-index fits were run twice for the two GCs exhibiting emission lines: once including all indices, and a second time excluding Balmer indices (which are probably suffering fill-in by emission lines). The best-fit GC parameters do not change significantly between the two runs.

\section{Abundance Ratios}

Are old GCs in other galaxies $\alpha$-enhanced, like Milky Way globular clusters? We should be able to assess $[\alpha / \mathrm{Fe}]$ for NGC 7457 GCs using Lick indices, plotting an $\alpha$-element index (usually involving $\mathrm{Ca}$ or $\mathrm{Mg}$ ) against an iron-sensitive index. However, this task turns out to be difficult in reality, suffering from observational and theoretical uncertainties.

Figure 8 plots the Mg2 index against the mean of the Fe5270 and Fe5335 indices. SSP models for $[\alpha / \mathrm{Fe}]=0.0$ and $[\alpha / \mathrm{Fe}]=0.3$ (Thomas et al. 2003) are superimposed on the plot. The majority of GCs form a cloud with large scatter at low metallicities. It is difficult to reach conclusions about their abundance ratios due to the large observational errors, coupled with the convergence of the models at these low metallicities.

The $[\alpha / \mathrm{Fe}]$ values derived from the $\chi^{2}$-minimization multi-index fits, listed in Table 5 , are consistent with $\alpha$-element enhancement, although they have large errors. The weighted mean $[\alpha / \mathrm{Fe}]$ for all $13 \mathrm{GCs}$ is $0.3 \pm 0.1$. However, one of the metal-rich GCs with emission lines (GC7) appears more consistent with $[\alpha / \mathrm{Fe}]=0$ than $[\alpha / \mathrm{Fe}]=0.3$. GC5 appears to have a low $[\alpha / \mathrm{Fe}]$ in Figure 8 , but its multi-line fit gives $[\alpha / \mathrm{Fe}]=0.3 \pm 0.2$.

The center of NGC 7457 itself clearly has a near-solar abundance ratio. It sits firmly on the $[\alpha / \mathrm{Fe}]=0.0$ model track in Figure 8, and the multi-index fitter gives $[\alpha / \mathrm{Fe}]=-0.03 \pm 0.03$.

\section{Ages and Metallicities}

We can break the age-metallicity degeneracy for our GCs by plotting an age-sensitive index (a Balmer line) against a metallicity-sensitive index (such as Fe5270, Fe5335, or Mg2). In Figure 9, we plot $\mathrm{H} \beta$ against $<\mathrm{Fe}>$. Overplotted on our data are theoretical isochrone and isometallicity lines from Thomas et al. (2003) which assume $[\alpha / \mathrm{Fe}]=0.3$. In this plot, all of our globular clusters appear old. They cluster around the $14 \mathrm{Gyr}$ isochrone, but it is difficult to draw conclusions about their ages beyond the fact that they are $>5$ Gyr old. The multi-index fits listed in Table 5 also imply old ages for the GCs. The weighted mean age for the 11 GCs which do not exhibit emission lines is $11.2 \pm 1.6$ Gyr.

There is striking contrast between the globular clusters and the central region of NGC 7457 itself. The galaxy's nucleus is much younger; in Figure 9, it appears to be dominated by stars aged 1.7 Gyr. However, keep in mind that the $\alpha$-enhanced models are not appropriate for comparison 
with the galaxy spectrum, as the center of NGC 7457 exhibits $[\alpha / \mathrm{Fe}]=0$. The multi-index fit agedates the center of NGC 7457 as $2.5 \pm 0.3 \mathrm{Gyr}$ old, in agreement with the observations of Sil'chenko et al. (2002), who assigned an age of 2-2.5 Gyr.

The galactic nucleus is also significantly more metal-rich than even the most enhanced GC. Its multi-index fit implies a super-solar metallicity of $[\mathrm{Fe} / \mathrm{H}]=0.18$. This is more metal-rich than the solar metallicity found for NGC 7457's nucleus by Sil'chenko et al. (2002), but this discrepancy is probably only a difference in models used; Sil'chenko et al. measure similar Lick index values, but they compare their data with models from Worthey (1994).

As seen in Figures 9 and 10, the GCs themselves occupy a broad spread in metallicity, as the GCs extend from low metallicities to near-solar metallicities. No bimodality is apparent in these figures, and should not be expected due to small sample size and inherent biases in our selection of spectroscopic candidates. The multi-index fits imply $[\mathrm{Fe} / \mathrm{H}]$ values in the range of -1.6 to -0.3 , consistent with the composite metallicities.

The two GCs exhibiting emission lines (ELGCs), GC6 and GC7, are by far the most metalrich GCs in our sample. Their metallicities are almost solar, with multi-index fits giving $[\mathrm{Z} / \mathrm{H}]$ of $-0.1 \pm 0.1$ and $-0.2 \pm 0.2$. As seen in Table 3, they are also the most metal-rich GCs according to their composite metallicities, and have composite $[\mathrm{m} / \mathrm{H}]$ quite similar to that of the center of NGC 7457. One must note the significant caveat that these composite metallicities are calibrated using Galactic GCs, and so one should expect systematic deviations for young ages and solar abundance ratios. When we take into account all the different methods for measuring metallicity, it appears that GC6 and GC7 may have metallicities which are closer to the center of NGC 7457 than to the 11 normal GCs.

Additionally, GC6 and GC7 are possible exceptions to the norm of old GC ages. Their Balmer absorption lines are being filled in by emission, and therefore our Balmer index measurements should be seen as lower limits. They appear to follow similar trends as the rest of the GCs in the $\mathrm{H} \beta$ against $<\mathrm{Fe}>$ plot, but we can also assess their ages using a higher-order Balmer line, which should suffer less emission line fill-in.

Figure 10 plots $\mathrm{H} \delta \mathrm{F}$ against $<\mathrm{Fe}>$. Despite larger errors for $\mathrm{H} \delta \mathrm{F}$, the two ELGCs (seen as open triangles) sit near the galactic center in this parameter space. All three objects have supersolar metallicity in this plot. There are hints that the two ELGCs may have intermediate ages like the galactic center ( $\sim 4-5$ Gyr according to this plot).

A significant body of evidence therefore supports a scenario where the stellar populations of the two ELGCs resemble the luminosity-weighted galaxy center more closely than they resemble the other normal GCs in the NGC 7457 system. However, the multi-index fits to GC6 and GC7 age-date them as old (11-15 Gyr), both when we include and exclude the Balmer indices. Of course, the metal-index Lick indices are primarily sensitive to metallicity, but they retain some sensitivity to age. To our knowledge, deriving ages solely from metal lines has not been attempted for objects known to be of intermediate age, so it is unclear how significant the multi-index derived old ages 
for GC6 and GC7 are.

\section{Two Emission Line Objects in GCs}

\subsection{Association between GCs and the Emission Line Sources}

Both ELGCs are part of the NGC 7457 system. The radial velocities of GC6 $\left(818 \pm 11 \mathrm{~km} \mathrm{~s}^{-1}\right)$ and GC7 $\left(822 \pm 10 \mathrm{~km} \mathrm{~s}^{-1}\right)$ are consistent with the radial velocity of the galaxy itself $(789 \pm 15 \mathrm{~km}$ $\mathrm{s}^{-1}$ ), confirming these objects as members of the NGC 7457 system. Additionally, GC6 and GC7 have very similar radial velocities to one other.

The sources of the emission lines appear kinematically coupled to the clusters. In the case of GC6, the [O III] $\lambda \lambda 4959,5007$ lines have a radial velocity of $857 \mathrm{~km} \mathrm{~s}^{-1}$, while the stellar absorption associated with this cluster has a radial velocity of $818 \pm 11 \mathrm{~km} \mathrm{~s}^{-1}$. The [O III] lines in GC7 have a radial velocity of $837 \mathrm{~km} \mathrm{~s}^{-1}$, in comparison with the stellar spectrum of the cluster receding

at $822 \pm 10 \mathrm{~km} \mathrm{~s}^{-1}$. Our measurements of the emission line radial velocities are only based on two lines, and therefore will have rather large errors. Our data therefore imply that, within the errors, the emission line sources are associated with the clusters.

As can be seen in Figure 11, which shows our two dimensional spectra for GC6 and GC7 around $5000 \AA$, the bright [O III] emission is spatially contained within the clusters. In both clusters, there is some hint of diffuse [O III] $\lambda 5007$ emission, but at a significantly higher velocity than the concentrated emission in the clusters. (This may be the diffuse [O III] emission observed at $985 \mathrm{~km} \mathrm{~s}^{-1}$ with SAURON integral field unit spectroscopy by Sarzi et al. 2006.) We therefore conclude that the sources of the [O III] emission are within GC6 and GC7.

\subsection{ELGC Morphology}

Interestingly, GC6 and GC7 are adjacent to one another on the sky, with a projected separation of $13.6^{\prime \prime}$ ( $\sim 850 \mathrm{pc}$ at a distance of $13 \mathrm{Mpc}$ ). Their similar positions and radial velocities could suggest that these two GCs are on similar orbits around the galaxy. Their positions can be seen in Figure 12, an HST/WFPC2 image of NGC 7457. The inset details in Figure 12 show GC6 and GC7 at WFPC2 resolution, along with normal globular cluster GC8.

Some asymmetry is obvious for GC6, with the cluster appearing as an unresolved source with an extended tail in both the $\mathrm{F} 555 \mathrm{~W}$ and $\mathrm{F} 814 \mathrm{~W}$ bands. It is possible that this $\mathrm{GC}$ is superimposed on a background object, with a more distant galaxy appearing as the tail. However, with an upper

limit to its size of $\mathrm{R}_{e f f} \lesssim 1$ pc given by our ishape analysis, the concentrated knot of GC6 is much smaller than typical GCs, usually 3-4 pc (Larsen 2004; Jordán et al. 2005).

On the other hand, GC7 has an unusually large effective radius, 12.3 pc. Curiously, Larsen \& 
Richtler (2006) find similarly large sizes for the clusters in their sample which host emission-line objects, though these are all young clusters with ages $<1$ Gyr.

The luminosities of these two ELGCs are typical of globular clusters. GC7 has an absolute V magnitude of -8.2 , and GC6 has $\mathrm{M}_{V}=-8.1$. This is less than $\sim 1$ magnitude brighter than the turnover magnitude for the Milky Way GC luminosity function (GCLF) $\left(\mathrm{M}_{V}=-7.4\right)$, and is well within $1 \sigma$ (1.2 mag; Harris 2001) of the peak of a typical log-normal GCLF.

\subsection{Emission Line Spectra}

The $[\mathrm{O}$ III $] \lambda \lambda 4959,5007$ emission lines dominate the emission-line spectra for both ELGCs. However, a few other emission lines become apparent in GC6 and GC7 after closer inspection. In order to isolate the emission lines, the GC spectra have been normalized, and a MILES SSP model was subtracted from them. Because of the issues described in Section 5, we can not be sure which SSP would be the best fit to GC6 and GC7. Therefore, throughout our analysis, we compared GC6 and GC7 with two MILES models: one was a model with solar metallicity and an age of 2.0 Gyr, in order to mimic the stellar population in the nucleus of NGC 7457, while the other was typical of a metal-rich $\mathrm{GC}$, with an age of $12.6 \mathrm{Gyr}$ and metallicity $[\mathrm{M} / \mathrm{H}]=-0.38$. The reader should note that our choice of model does not affect our measurements of [O II] and [O III] much, but does impact our results for $\mathrm{H} \beta$. It is also important to note that the sensitivity of the spectrograph drops significantly at blue wavelengths, so our measurements of [O II] suffer from low signal-to-noise.

We require models of the underlying stellar population for two reasons. First, in order to account for any absorption underlying the emission lines, we measure the MILES equivalent width and the GC equivalent width over the same bandpass, and take the difference of the two to determine the total equivalent width of the emission line. Secondly, we use the MILES models to constrain the overall shape of the continuum, so that when we compare line strengths at $4861 \AA / 5007 \AA$ with those at $3727 \AA$, we will not be affected by dust extinction or errors in the flux calibration.

Table 6 lists the qualitative strengths of lines which are seen in emission after MILES SSP models are subtracted from these two objects. GC7 is a high-excitation nebula, with no observable [O II] emission, but very strong [O III] emission $\left(\mathrm{L}_{5007}=(1.2 \pm 0.3) \times 10^{36} \mathrm{erg} \mathrm{s}^{-1}\right)$, and visible [Ne III] emission. Unfortunately, we were not able to derive nebular parameters for this object, because the Balmer emission and lines produced by other ionization states of oxygen were not strong enough to measure (see Figure 13).

GC6 is a much lower excitation nebula than GC7, with significant [O II] emission and weaker [O III] emission $\left(\mathrm{L}_{5007}=(2.4 \pm 0.7) \times 10^{35} \mathrm{erg} \mathrm{s}^{-1}\right)$. The [O II] $\mathrm{H} \beta$, and [O III] emission lines can be seen for both clusters in Figure 13, with the solar metallicity, 2.0 Gyr MILES model subtracted from them. The emission-line ratios for GC6 are listed in Table 7. Two values are listed for each ratio: one utilizing the 2.0 Gyr MILES model, and one which uses the 12.6 Gyr old model. As we will discuss in the next section, these line ratios for GC6 are difficult to explain in an old stellar 
population.

\subsection{The Nature of the Emission Line Sources}

Any thorough picture of the GC system of NGC 7457 must take these peculiar emission-line objects into account, but we are forced to admit that there is no simple explanation for them. In extragalactic systems, a compact source of strong [O III] emission is often assumed to be a planetary nebula (PN). However, PNe have proven elusive in GCs, and are still poorly understood. We must consider all the possible options for sources that might power the observed emission lines.

In the Milky Way, only four PNe have been detected in 133 GCs surveyed (Jacoby et al. 1997). Meanwhile, in the large samples of extragalactic GC spectra which now exist in the literature, only a small handful of ELGCs have been found. Little effort has been spent characterizing the sources of the observed emission lines. Three emission-line objects were found in GCs in the giant

elliptical galaxy NGC 5128 (Minniti \& Rejkuba 2002; Rejkuba et al. 2003), and were assumed to be PNe. Brodie et al. (2005) identified one ELGC in their spectroscopic sample of 22 GCs in the elliptical galaxy NGC 4365, while in NGC 3379, Pierce et al. (2006) found that two of their 22 spectroscopically-observed GCs hosted emission line objects. Larsen \& Richtler (2006) performed a detailed study of the [O III] $\lambda \lambda 4959,5007$ and [N II] $\lambda \lambda 6548,6584$ emission they found in three young star clusters (aged between $30 \mathrm{Myr}$ and $280 \mathrm{Myr}$ ) in the spiral galaxies NGC 5236 and NGC 3621. Finally, Zepf et al. (2007) recently found [O III] emission in a NGC 4472 GC, and identified its source as a black hole X-ray binary.

\subsubsection{Planetary Nebulae?}

It is difficult to constrain the ionization source creating the emission lines in GC7, as it lacks measurable $[\mathrm{O}$ II] $\lambda 3727$ and $\mathrm{H} \beta$. However, the presence of [Ne III] and strong [O III] are typical of PNe (Jacoby \& Ciardullo 1999). This source has an [O III] absolute magnitude of $\mathrm{M}_{5007}=$ -3.7 , as defined in Ciardullo et al. (2005). This is a typical value for a PN, 0.8 magnitudes fainter than the PN luminosity function cut-off. We can get a rough lower limit for the mass of the central star if we can derive lower limits for the luminosity and temperature of the central star. We constrain luminosity by assuming that no more than $10 \%$ of the central star's flux can emerge as the $[\mathrm{O} \mathrm{III}] \lambda 5007$ line (an assumption supported by both theoretical and observational findings: Ciardullo et al. 2005). Therefore, the central star of the PN in GC7 has a minimum luminosity of $3080 \mathrm{~L}_{\odot}$. Additionally, we can place a lower limit on the effective temperature of the central star if we can calculate E, the excitation parameter which is defined by Dopita et al. (1992) as $\mathrm{E}=0.45$ ( [O III $] \lambda 5007 / \mathrm{H} \beta$ ). This formulation for $\mathrm{E}$ is only valid if $\mathrm{E} \leq 5.0$; to constrain greater values of $\mathrm{E}$, a flux for the He II $\lambda 4686$ line must be measured. Although we can not confidently measure $\mathrm{H} \beta$, we can place upper limits on its flux, giving [O III $] \lambda 5007 / \mathrm{H} \beta \gtrsim 15$, 
and clearly implying $\mathrm{E}>5.0$. Although we can not actually measure $\mathrm{E}$ because the He II line was not observable, we can use this lower limit on E to derive a lower limit on the central star's temperature. Using equation 2.2 in Dopita et al. (1992), we find that $\log \left(\mathrm{T}_{\text {eff }}\right)>5.0$.

As can be seen in Figure 14, which plots Vassiliadis \& Wood (1994) model tracks for post-AGB evolution, these modest lower limits on the central star's luminosity and temperature imply a mass

$>0.58 M_{\odot}$, corresponding (in these models) to a progenitor main sequence mass $>1.1 \mathrm{M}_{\odot}$. These models are for approximately solar metallicity; lower metallicity model tracks shift slightly upward, and the inferred lower limit to main sequence mass would be $\sim 1 \mathrm{M}_{\odot}$ for $[Z / \mathrm{H}]=-0.6$. In a solar metallicity population, a turn-off mass of $1.1 \mathrm{M}_{\odot}$ corresponds to an age of 7 Gyr, while in a typical metal-poor GC, a $1.0 \mathrm{M}_{\odot}$ turns off at this age (Demarque et al. 2004); this implies that GC6 is $\leq 7$ Gyr old.

Intermediate ages are also supported by empirical studies; Jacoby \& Ciardullo (1999) derive nebular parameters from high-quality spectra for PNe in M33, and find that all the PNe in their sample with $\mathrm{M}_{5007}$ brighter than -3.0 have central star masses $\geq 0.6 \mathrm{M}_{\odot}$. The white dwarfs of mass $0.61 M_{\odot}$ in the solar metallicity open cluster NGC 7789 have progenitor masses of $2.0 M_{\odot}$ (Kalirai et al. 2007), so we would expect a minimum progenitor mass of $\sim 1.5-2.0 M_{\odot}$ in our case. Therefore, both the theoretical and empirical models indicate a progenitor main sequence mass of $>1 M_{\odot}$ for GC7. If GC7 is an old GC, the progenitor must be more massive than the main sequence turnoff stars - probably a blue straggler. If the progenitor is instead a normal turnoff star, then the corresponding cluster age depends upon the exact mass, with a maximum age of 7 Gyr and a more probable age of $\sim 2$ Gyr. Such ages are consistent with the luminosity-weighted age of the galaxy center.

On the other hand, the emission line spectrum of GC6 is of such low excitation that it would constitute a rather unusual PN. The near-equal strengths of [O II] and [O III] imply a cool central star $(\mathrm{T} \approx 36,500 \mathrm{~K})$, implying that much less than $10 \%$ of the central star's flux is observed as [O III]. Given the significant [O III] $\lambda 5007$ luminosity measured, one finds that the luminosity of the central star would have to be at least $3 \times 10^{4} \mathrm{~L} \odot$. These parameters imply a central star with a core mass of $>0.9 \mathrm{M}_{\odot}$ (Vassiliadis \& Wood 1994). As is apparent from Figure 14, the progenitor of such a central star would have been very massive on the Main Sequence- greater than $5 \mathrm{M}_{\odot}$. Such a massive star is difficult to explain in any but the youngest stellar populations. A blue straggler of such mass is extraordinarily unlikely. Therefore, the emission line source in GC6 is most probably not a PN.

\subsubsection{Supernova Remnants?}

CJFN 470 is an emission line source in M31 which closely resembles that in GC6, and was classified as a PN by Jacoby \& Ciardullo (1999). It is the only source in their sample with approximately equal strengths of $[\mathrm{O}$ II $] \lambda 3727$ and $[\mathrm{O}$ III $] \lambda 5007$. A closer look at this source reveals $[\mathrm{S}$ II] 
lines which are nearly as strong as $\mathrm{H} \alpha$ - a strong indicator that this object is a SNR.

Bright lines from lower ionization states are expected if the nebula is shock-heated, and not photoionized. Supernova remnants (SNRs) are often selected by their relatively bright [S II] emission (as compared with $\mathrm{H} \alpha$ ), and display high levels of [O I] and [O II] emission as well (Fesen et al. 1985). It is probable that GC6 hosts a SNR, although it is difficult to be certain without spectral coverage of the $[\mathrm{S} \mathrm{II}] \lambda \lambda 6717,6731$ and $[\mathrm{O} \mathrm{I}] \lambda \lambda 6300,6363$ lines.

A detection of an SNR in a GC by its [O III] lines is not unprecedented. Minniti \& Rejkuba (2002) asserted that they had found the first PN in a GC in an elliptical galaxy. However, with the higher-resolution spectroscopy of Peng et al. (2004), the [O III] emission lines were observed to be

double-peaked, with the peaks separated by $\sim 300 \mathrm{~km} \mathrm{~s}^{-1}$. The authors note that such kinematics are implausible for a PN, which usually have maximum expansion velocities of a few times $10 \mathrm{~km}$ $\mathrm{s}^{-1}$.

There is no evidence for similarly high expansion velocities in our emission line objects. In both cases, the [O III] lines are unresolved, placing an upper limit on their velocity widths of $165 \mathrm{~km}$ $\mathrm{s}^{-1}$. However, this upper limit does not exclude the possibility of GC6 hosting an SNR. The oxygen lines in the spectrum imply that the SNR is in an evolved, radiative stage with emission arising from swept-up shocked ISM. Typical shock velocities for this stage are $50-200 \mathrm{~km} \mathrm{~s}^{-1}$ (Weiler \& Sramek 1988).

Maoz et al. (2005) point out that an SNR in a very low-density medium will only glow as a non-radiative Balmer-dominated SNR, and once it has expanded through all of its circumstellar material, it will fade quickly. Therefore, the presence of radiative SNRs in GCs around early-type galaxies can place constraints on the ISM density in these clusters. Future observations with better velocity resolution and extended wavelength coverage will allow us to model the gas density in GCs.

This SNR probably does not explain the curious morphology of GC6 as it is quite evolved. The optical continuum emission of a SN Type Ia fades quickly, falling to the R-band luminosity of GC6 2-3 years after the explosion, and to 10\% the luminosity of GC6 less that one year after that (Woosley et al. 2007, Lair et al. 2006).

Although an unrelated bright point source might contribute to the compact profile of the cluster and the illusion of a lower-surface brightness tail, we can exclude transient phenomena as the cause of GC6's morphology. The flux of GC6 does not appear to be time-variable, as the spectroscopic continuum flux of GC6 in our 2003 observations is approximately the same as or other GCs which had similar V magnitudes as GC6 in the Cycle 4 HST images.

\subsubsection{Binary Stars?}

Several kinds of binary star systems can produce bright [O III] emission in intermediate-to-old stellar populations. One such breed of binary system are symbiotic stars (SSs), which feature a 
hot component (usually a white dwarf), and a cool component (an evolved giant). The dense wind from the giant is irradiated with UV emission from the white dwarf, producing emission lines which can closely mimic those in PNe. Within the Local Group, SSs can be differentiated from PNe by the underlying red continuum from the giant. However, at distances beyond the Local Group, the continuum from this single star becomes too faint to detect (Magrini et al. 2003). Additionally, emission-line sources in GCs are surrounded by stars, which overwhelm any stellar giant companion to a white dwarf. We must therefore use emission line ratios to distinguish PNe from SSs.

Gutiérrez-Moreno et al. (1995) considered such spectral diagnostics, and determined that SSs do not show $[\mathrm{O} \mathrm{III}] \lambda 5007 / \mathrm{H} \beta \gtrsim 10$. Objects with $[\mathrm{O}$ III $] / \mathrm{H} \beta<10$ may be either SSs or PNe. As discussed in the last section, for GC7 we know that $[\mathrm{O} \mathrm{III}] / \mathrm{H} \beta>15$. Therefore, GC7 probably hosts a PN.

We can not use this diagnostic for GC6, because its $[\mathrm{O}$ III $] / \mathrm{H} \beta=2.3-3.9$, well within the regime inhabited by both PNe and SSs. However, diagnostic diagrams developed in Schwarz (1988) and Gutiérrez-Moreno (1988) make use of [O II] $\lambda 3727$, [O III $] \lambda 5007$, and $\mathrm{H} \beta$, and are useful for constraining the nature of GC6. These diagrams employ the $\Delta \mathrm{E}$ parameter defined in Baldwin et al. (1981), where:

$$
\Delta E=\log ([\mathrm{O} \mathrm{III}] / H \beta)+\log (([\mathrm{O} \mathrm{II}] /[\mathrm{O} \mathrm{III}])+0.32)-0.44 .
$$

We measure values of $\Delta \mathrm{E}=0.1-0.3$ for GC6 (depending on which SSP model is subtracted). Symbiotic Stars with similar [O II]/[O III] as GC6 have $\Delta \mathrm{E}<-0.15$, so it is clear that GC6 does not contain a SS. Curiously, GC6 sits comfortably in the H II region zone of the Schwarz (1988) diagnostic plot. However, we dismiss this option because our measured absorption-line spectrum clearly shows that GC6 does not contain young $(<10$ Myr) stars.

Finally, a binary system containing a compact object can generate optical emission lines (Zepf et al. 2007). We examined archival Chandra X-ray Observatory data for NGC 7457, and concluded that there are no X-ray sources coincident with GC6 or GC7. Additionally, the low velocity dispersions of the emission lines in these clusters would be unusual for a binary system containing a neutron star or black hole (for contrast, see the broad velocity structure of the [O III] lines in Zepf et al. 2007).

We can therefore exclude the possibility of GC6's emission coming from a PN, an SS, an X-ray binary, or an H II region; the emission lines are probably coming from an SNR.

\section{Discussion and Conclusions}

Together, these analyses strongly suggest a significant population of intermediate-age GCs in NGC 7457. Although no one piece of evidence is conclusive, there is a large body of data

hinting towards peculiar stellar populations in this GC system. In review, the evidence suggesting intermediate-age GCs includes: 
1. V-I color-magnitude diagram. Even with HST photometry, the distribution of GC colors appears broad, and does not have two subpopulations at the "usual" locations. A simple explanation for this is an intermediate-age stellar population filling in the gap between the two normal old GC subpopulations.

2. Color-color analysis. The location of several objects in the $F 330 W-V$ vs. $V-I$ plot are consistent with intermediate ages, but do not require them.

3. High metallicities of GC6 and GC7. The metallicities of these two clusters are significantly higher than any of the other GCs, and in many of our measurements they appear more similar to the galactic nucleus than to typical metal-rich GCs.

4. Strong higher-order Balmer line absorption for GC6 and GC7. H $\delta$ F should be less affected by emission-line fill-in than $\mathrm{H} \beta$, and GC6 and GC7 are found near the 2.5 Gyr-old galactic nucleus in an $\mathrm{H} \delta \mathrm{F}-<\mathrm{Fe}>$ line diagnostic plot.

5. Significant evidence for a $>1 \mathrm{M}_{\odot}$ progenitor for the PN in GC7. Although such a progenitor could have been a blue straggler in an old stellar population, it would be simpler to explain it as a normal main sequence star in a $\sim 2-3$ Gyr old stellar population.

6. A probable SNR in GC6. Given the intermediate-to-old nature of the GC stellar population, the SN was almost certainly Type Ia. Various models featured in Matteucci \& Recchi (2001) predict that SNe Ia rates are anywhere between 7 and 158 times higher in a 2.5 Gyr old stellar population as compared to a 12 Gyr old stellar population.

All together, this evidence makes a strong case for the existence of at least some intermediateage GCs, which trace the star-formation event that dominates the stellar population in the center of NGC 7457. The total number of such GCs is uncertain. Two out of 13 spectroscopic GCs may be of intermediate age, while photometry suggests that a third or more of the GCs in the WFPC2 frame could be of intermediate-age. Much larger spectroscopic samples would be required to determine the fraction conclusively.

It is important to remember that the two near-universal GC subpopulations of old metal-rich and old metal-poor stars are present in NGC 7457. There are significant numbers of GCs at the appropriate colors predicted by the GC color-galaxy luminosity relation. Meanwhile, eleven out of 13 spectroscopic GCs appear old, with metallicities ranging from $[\mathrm{Fe} / \mathrm{H}]=-0.8$ to -1.8 .

The kinematics of the GCs show rotation around the photometric minor axis of the galaxy. The orientation of the rotation is generally consistent with that of the galaxy itself, as determined by integral-field spectroscopy.

What general conclusions may be drawn about the history of this unusual S0? NGC 7457 has been seen as a classic example of a pseudobulge (that is, a disky bulge built through secular processes) due to its very low velocity dispersion. The existence of a significant number of old, 
metal-rich GCs - usually associated with merger-built "classical" bulges - indicates that the bulge of NGC 7457 is unlikely to have been built solely through secular means at relatively recent times. A number of GCs appear to have been added in a recent star-forming event that dominates the stellar population of the most central regions; therefore, the event probably involved a substantial amount of gas.

Even the younger GCs need not be associated with a pseudobulge. The two ELGCs with presumptive intermediate-ages are located at projected radii of $32^{\prime \prime}$ and $44^{\prime \prime}$ (2.0 and $\left.2.8 \mathrm{kpc}\right)$. The bulge itself dominates the light profile only within $9^{\prime \prime}$ (J. Kormendy 2008, private communication), and it is not clear that purely secular star formation would lead to GC formation at larger radii.

A larger kinematic and stellar population study of the metal-rich GCs in NGC 7457 would help illuminate the relative importance of rotation and pressure support among the old and intermediateage GCs, and thus whether a secular or merger origin is more likely.

We have also shown that it is critical to acknowledge and understand the presence of emission lines in spectroscopic GC samples, as emission can strongly affect some lines (such as Balmer lines) through fill-in and thus change the derived cluster ages. Our analysis has also demonstrated the utility of emission line objects as an alternate route to studying the stellar populations of extragalactic GCs. While we did not have the necessary emission lines in this study to calculate chemical abundances, future studies with higher $\mathrm{S} / \mathrm{N}$ spectra and wider wavelength coverage could estimate abundances of light elements like $\mathrm{O}, \mathrm{N}$, and $\mathrm{S}$ that are difficult to derive from absorptionline spectra.

\section{Acknowledgments}

We are very grateful to Jay Gallagher, George Jacoby, John Kormendy, and Nick Konidaris for useful discussions and we thank our referee, Jimmy Irwin, for his helpful suggestions. We are indebted to Rob Proctor for insight into his multi-index fitter. We also thank Javier Cenarro, Glenda Denicoló, Mike Beasley, and Duncan Forbes for their help with the data reduction. We acknowledge support from the National Science Foundation through grants AST 0206139 and AST 0507729. Support for this work was provided by NASA through Hubble Fellowship grant HF01214.01-A awarded by the Space Telescope Science Institute, which is operated by the Association of Universities for Research in Astronomy, Inc., for NASA, under contract NAS 5-26555.

This research has made use of the NASA/IPAC Extragalactic Database (NED) which is operated by the Jet Propulsion Laboratory, California Institute of Technology, under contract with the

National Aeronautics and Space Administration. The SSC Spitzer Tools also proved useful to this research. Finally, we acknowledge the usage of the HyperLeda database (http://leda.univ-lyon1.fr). 


\section{REFERENCES}

Baldwin, J. A., Phillips, M. M., \& Terlevich, R. 1981, PASP, 93, 5

Beasley, M. A., Strader, J., Brodie, J. P., Cenarro, A. J., \& Geha, M. 2006, AJ, 131, 814

Brodie, J. P., \& Strader, J. 2006, ARA\&A, 44, 193

Brodie, J. P., Strader, J., Denicoló, G., Beasley, M. A., Cenarro, A. J., Larsen, S. S., Kuntschner, H., \& Forbes, D. A. 2005, AJ, 129, 2643

Bruzual, G., \& Charlot, S. 2003, MNRAS, 344, 1000

Burstein, D., Faber, S., Gaskell, C., \& Krumm, N. 1984, ApJ, 287, 586

Cappellari, M. et al. 2006, MNRAS, 366, 1126

Cardiel, N., Gorgas, J., Cenarro, J., \& González, J. J. 1998, A\&AS, 127, 597

Cenarro, A. J., Beasley, M. A., Strader, J., Brodie, J. P., \& Forbes, D. A. 2007, AJ, 134, 391

Chapelon, S., Buat, V., Burgarella, D., \& Kissler-Patig, M. 1999, A\&A, 346, 721

Ciardullo, R., Sigurdsson, S., Feldmeier, J. J., \& Jacoby, G. H. 2005, ApJ, 629, 499

Demarque, P., Woo, J., Kim., Y., \& Yi, S. K. 2004, ApJS, 155, 667

de Vaucouleurs, G., de Vaucouleurs, A., Corwin Jr., H. G., Buta, R. J., Paturel, G., \& Fouque, P. 1991, Third Reference Catalogue of Bright Galaxies Version 3.9

Dolphin, A. E. 2000, PASP, 112, 1383

Dopita, M. A., Jacoby, G. H., \& Vassiliadis, E. 1992, ApJ, 389, 27

Dressler, A., \& Sandage, A. 1983, ApJ, 265, 664

Emsellem, E., et al. 2004, MNRAS, 352, 721

Fesen, R. A., Blair, W. P., \& Kirshner, R. P. 1985, ApJ, 292, 29

Fritze-v. Alvensleben, U., \& Burkert, A. 1995, A\&A, 300, 58

Girardi, L., Bertelli, G., Bressan, A., Chiosi, C., Groenewegen, M. A. T., Marigo, P., Salasnich, B., \& Weiss, A. 2002, A\&A, 391, 195

Goudfrooij, P., Alonso, M. V., Maraston, C., \& Minniti, D. 2001, MNRAS, 328, 237

Gutiérrez-Moreno, A. 1988 in ASP Conf. Ser. 1, Progress and Opportunities in Southern Hemisphere Optical Astronomy. The CTIO 25th Anniversary Symposium, ed. V. M. Blanco \& M. M. Phillips (San Francisco: ASP), 12 
Gutiérrez-Moreno, A., Moreno, H., \& Cortés, G. 1995, PASP, 107, 462

Harris, W. E. 2001 in Saas-Fee Advanced Course 28, Star Clusters, ed. L. Labhardt \& B. Binggeli (Berlin: Springer-Verlag), 223

Holtzman, J. A., Burrows, C. J., Casertano, S., Hester, J. J., Trauger, J. T., Watson, A. M., \& Worthey, G. 1995, PASP, 107, 1065

Jacoby, G. H., \& Ciardullo, R. 1999, ApJ, 515, 169

Jacoby, G. H., Morse, J. A., Fullton, L. K., Kwitter, K. B., \& Henry, R. B. C. 1997, AJ, 114, 2611

Jordán, A., et al. 2005, ApJ, 634, 1002

Kalirai, J. S., Hansen, B. M. S., Kelson, D. D., Reitzel, D. B., Rich, R. M., \& Richer, H. B. 2007, ApJ, submitted, astro-ph/0706.3894

Kormendy, J. 1977, ApJ, 214 , 359

Kormendy, J., \& Illingworth, G. 1983, ApJ, 265, 632

Kormendy, J., \& Kennicutt, Jr., R. C. 2004, ARA\&A, 42, 603

Kundu, A. \& Whitmore, B. C. 2001, AJ, 121, 2950

Lair, J. C., Leising, M. D., Milne, P. A., \& Williams, G. G. 2006, AJ, 132, 2024

Larsen, S. S. 1999, A\&AS, 139, 393

Larsen, S. S. 2004, A\&A, 416, 537

Larsen, S. S., \& Brodie, J. P. 2000, AJ, 120, 2938

Larsen, S. S., \& Brodie, J. P. 2002, AJ, 123, 1488

Larsen, S. S., \& Richtler, T. 2006, A\&A, 459, 103

Magrini, L., Corradi, R. L. M., \& Munari, U. 2003 in ASP Conf. Ser. 303, Symbiotic Stars Probing Stellar Evolution, ed. R. L. M. Corradi, R. Mikolajewska \& T. J. Mahoney (San Francisco: ASP), 539

Maoz, D., Waxman, E., \& Loeb, A. 2005, ApJ, 632, 847

Maraston, C. 2005, MNRAS, 362, 799

Maraston, C., Greggio, L., \& Thomas, D. 2001, Ap\&SS, 276, 893

Maraston, C., \& Thomas, D. 2000, ApJ, 541, 126

Massey, P., \& Gronwall, C. 1990, ApJ, 358, 344 
Matteucci, F., \& Recchi, S. 2001, ApJ, 558, 351

Minniti, D., \& Rejkuba, M. 2002, ApJ, 575, L59

Minniti, D., Rejkuba, M., Funes, J. G., \& Kennicutt, Jr., R. C. 2004, ApJ, 612, 215

Oke, J. B., et al. 1995, PASP, 107, 375

Paturel, G., Petit, C., Prugniel, Ph., Theureau, G., Rousseau, J., Brouty, M., Dubois, P., \& Cambrésy, L. 2003, A\&A, 412, 45

Peletier, R. F., Balcells, M., Davies, R. L., Andredakis, Y., Vazdekis, A., Burkert, A., \& Prada, F. 1999, MNRAS, 310, 703

Peng, E. W., Ford, H. C., \& Freeman, K. C. 2004, ApJS, 150, 367

Pierce, M. et al. 2006, MNRAS, 366, 1253

Proctor, R. N., Forbes, D. A., \& Beasley, M. A. 2004, MNRAS, 355, 1327

Rejkuba, M., Minniti, D., \& Walsh, J. R. 2003 in ESO Astrophys. Symp. Extragalactic Globular Cluster Systems, ed. M. Kissler-Patig (Berlin: Springer-Verlag), 133

Richardson, S. \& Green, P. G. 1997, JR Statist. Soc. B, 59, 731

Sage, L. J., \& Welch, G. A. 2006, ApJ, 644, 850

Sánchez-Blázquez, P., et al. 2006, MNRAS, 371, 703

Sarzi, M., et al. 2006, MNRAS, 366, 1151

Schlegel, D. J., Finkbeiner, D. P., \& Davis, M. 1998, ApJ, 500, 525

Schwarz, H. E. 1988 in ASP Conf. Ser. 1, Progress and Opportunities in Southern Hemisphere Optical Astronomy. The CTIO 25th Anniversary Symposium, ed. V. M. Blanco \& M. M. Phillips (San Francisco: ASP), 355

Sil'chenko, O. K., Afanasiev, V. L., Chavushyan, V. H., \& Valdes, J. R. 2002, ApJ, 577, 668

Sirianni, M., et al. 2005, PASP, 117, 1049

Strader, J., Beasley, M. A. \& Brodie, J. P. 2007, AJ, 133, 2015

Strader, J., Brodie, J. P., \& Forbes, D. A. 2004, AJ, 127, 3431

Strader, J., Brodie, J. P., Forbes, D. A., Beasley, M. A., \& Huchra, J. P. 2003a, AJ, 125, 1291

Strader, J., Brodie, J. P., Schweizer, F., Larsen, S., \& Seitzer, P. 2003b, AJ, 125, 626

Strader, J., Brodie, J. P., Spitler, L., \& Beasley, M. A. 2006, AJ, 132, 2333 
Sugimoto, D., \& Makino, J. 1989, PASJ, 41, 1117

Thomas, D., Maraston, C., \& Bender, R. 2003, MNRAS, 339, 897

Thomas, D., Maraston, C., \& Korn, A. 2004, MNRAS, 351, L19

Trager, S., Worthey, G., Faber, S., Burstein, D., \& Gonzalez, J. 1998, ApJS, 116, 1

van den Bergh, S. \& Mackey, A. D. 2004, MNRAS, 354, 713

Vassiliadis, E., \& Wood, P. R. 1994, ApJS, 92, 125

Weiler, K. W., \& Sramek, R. A. 1988, ARA\&A, 26, 295

Welch, G. A., \& Sage, L. J. 2003, ApJ, 584, 260

Woosley, S. E., Kasen, D., Blinnikov, S., \& Sorokina, E. 2007, ApJ, 662, 487

Worthey, G., \& Ottaviani, D. L. 1997, ApJS, 111, 377

Worthey, G. 1994, ApJS, 95, 107

Worthey, G., Faber, S. M., González, J. J., \& Burstein, D. 1994, ApJS, 94, 687

Zepf, S. E, Maccarone, T. J., Bergond, G., Kundu, A., Rhode, K. L., \& Salzer, J. J. 2007, ApJ, in press 


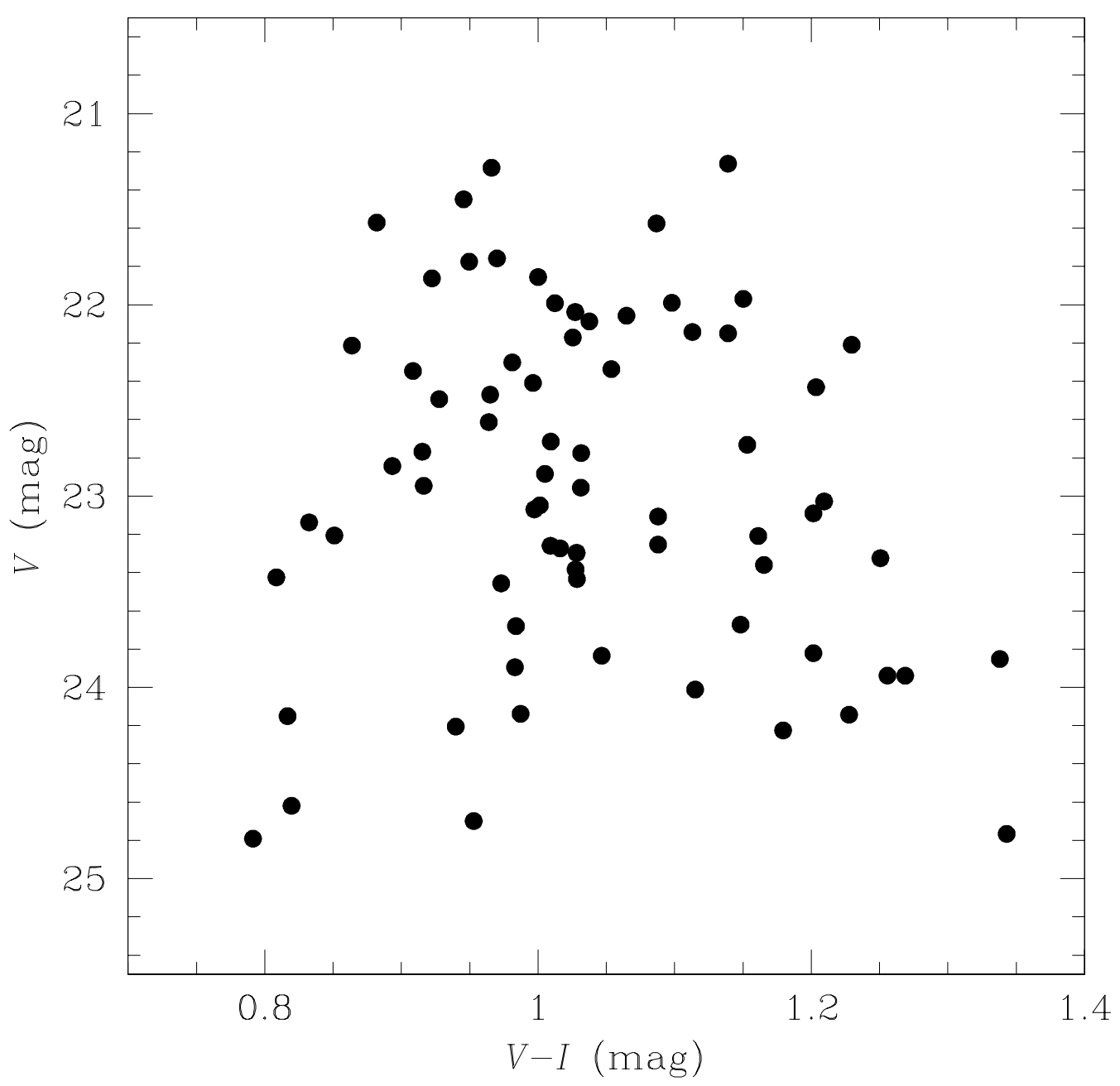

Fig. 1. - Color-magnitude diagram of GC candidates in NGC 7457 from VI HST/WFPC2 imaging. 


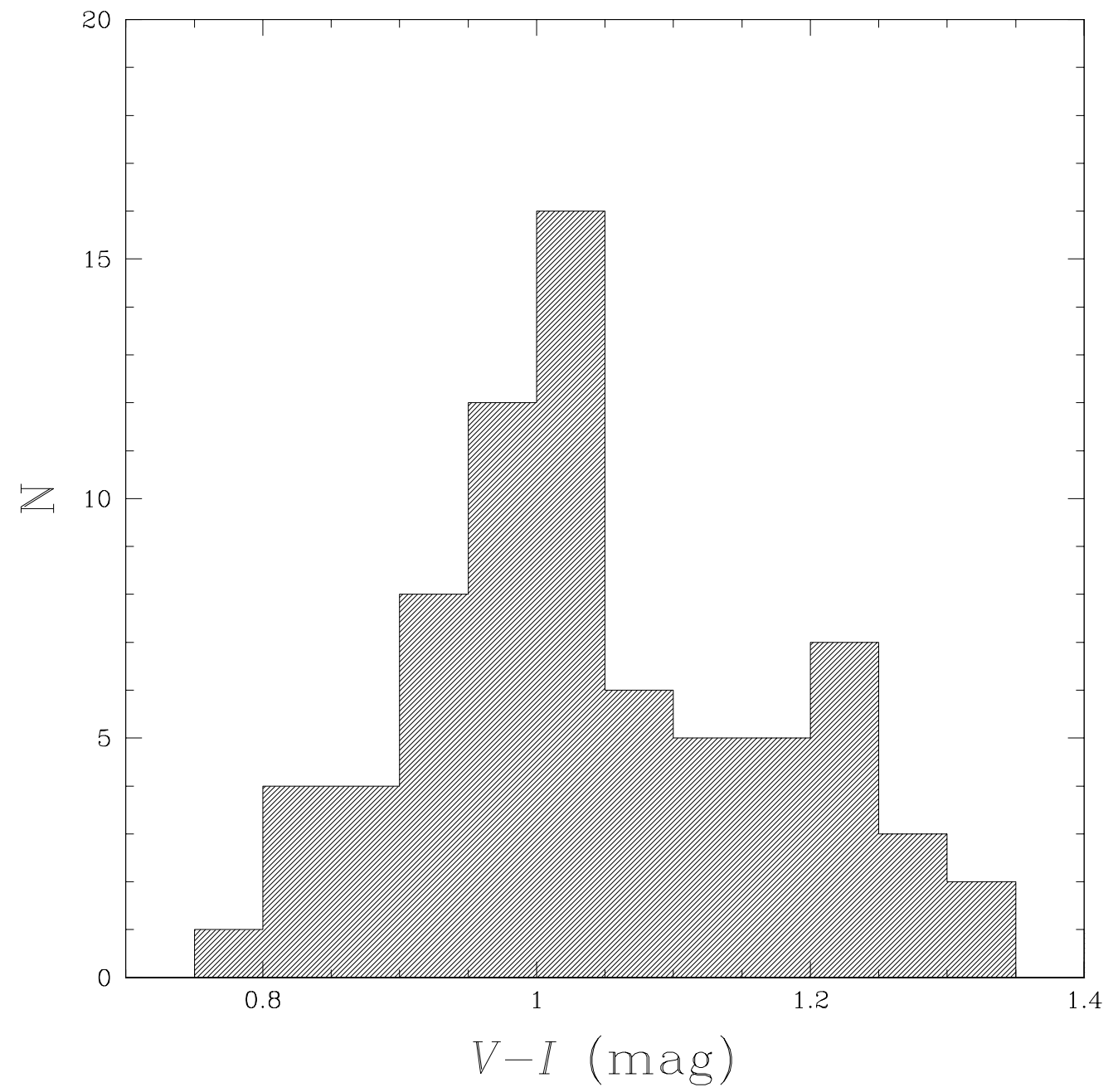

Fig. 2. $-V-I$ histogram of GC candidates. There is evidence for multiple subpopulations of GCs, and an enhancement of objects at $V-I \sim 1.02$ that is unusual for an early-type galaxy. 


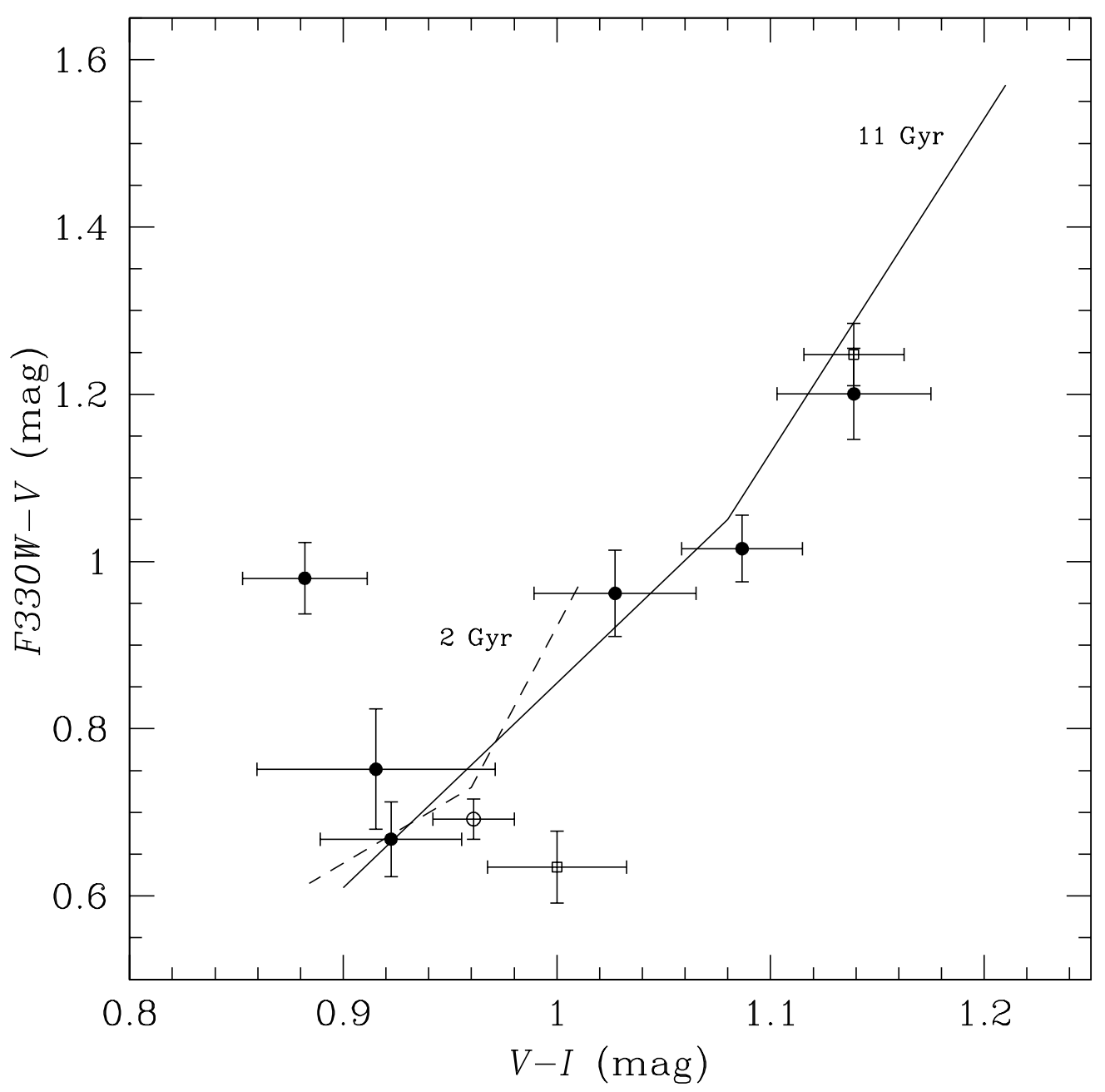

Fig. 3. $-F 330 W-V$ vs. $V-I$ color-color diagram of GC candidates. The open boxes are GCs with spectroscopic confirmation, the open circle is the large and luminous object L1; the filled circles are the remainder of the candidates. The dotted and solid lines are 2 and 11 Gyr isochrones from Girardi et al. (2002) with metallicities from $[Z / \mathrm{H}]=-1.7$ to 0 . A number of objects are too red to be of intermediate age, while several are consistent with either isochrone. 


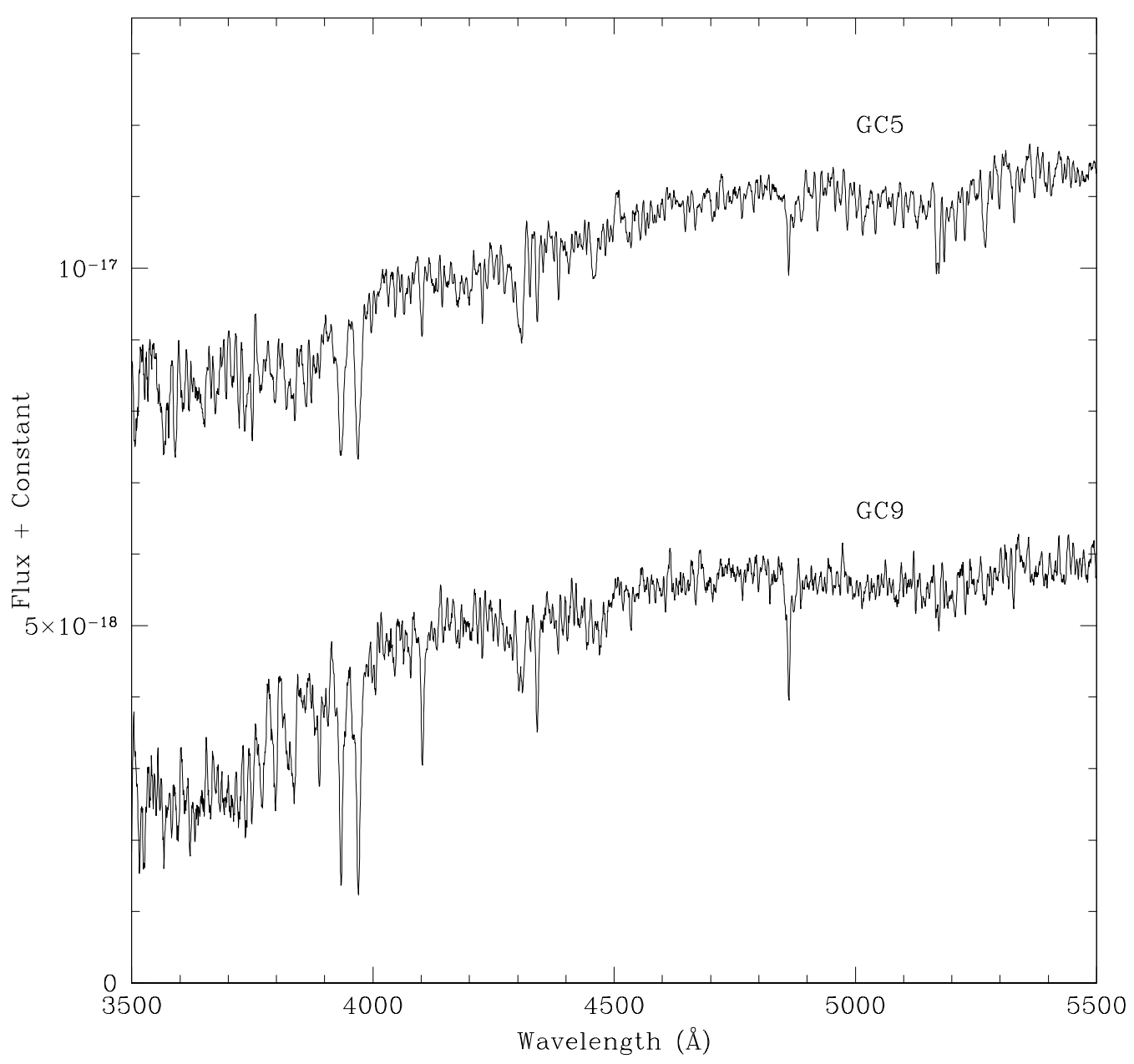

Fig. 4. - Spectra of metal-poor globular cluster GC9, and metal-rich globular cluster GC5. Notice the differences in strength of the Balmer line absorption (particularly $\mathrm{H} \beta$ at $4861 \AA$ and $\mathrm{H} \delta$ at $4102 \AA$ ) and the $\mathrm{Mg}$ band at $5170 \AA$. 


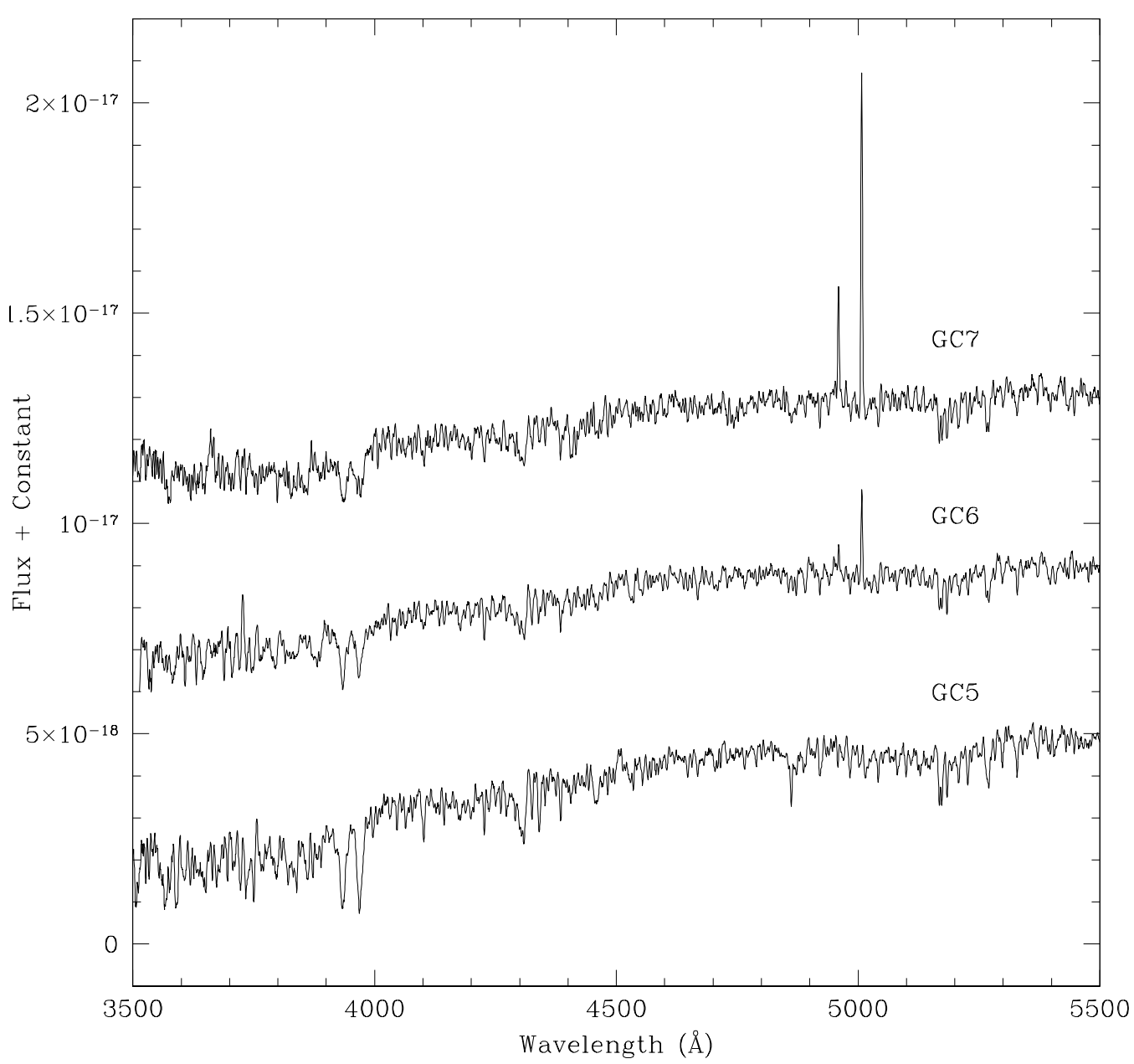

Fig. 5.- Spectra of GC6 and GC7, the two globular clusters with bright [O III] $] \lambda \lambda 4959,5007$ emission lines. Normal metal-rich globular cluster GC5 is included for comparison. GC6 features [O II] $\lambda 3727$ emission, and both GC6 and GC7 lack Balmer absorption due to fill-in by emission lines. 


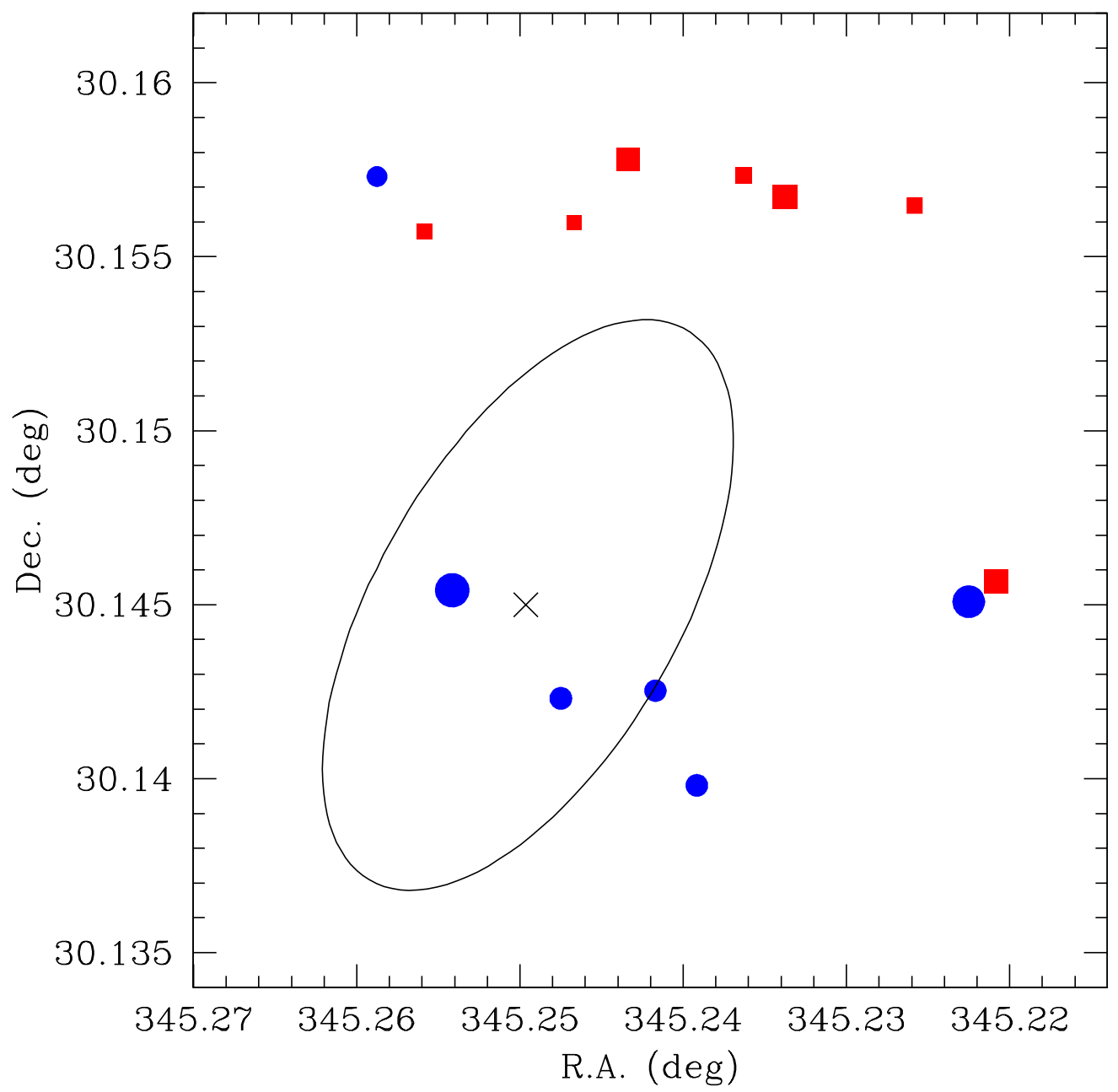

Fig. 6.- Kinematics of GCs in NGC 7457. Blue circles are GCs with radial velocities less than the median for the system; red squares have velocities larger than the median. The size of the point is proportional to the deviation from the median velocity. The center of the galaxy is marked with a cross, and a representative photometric isophote is plotted. Despite the unusual spatial distribution of the GCs, the general impression is that the GC system is rotating around an axis similar to that of the photometric minor axis of the galaxy. 

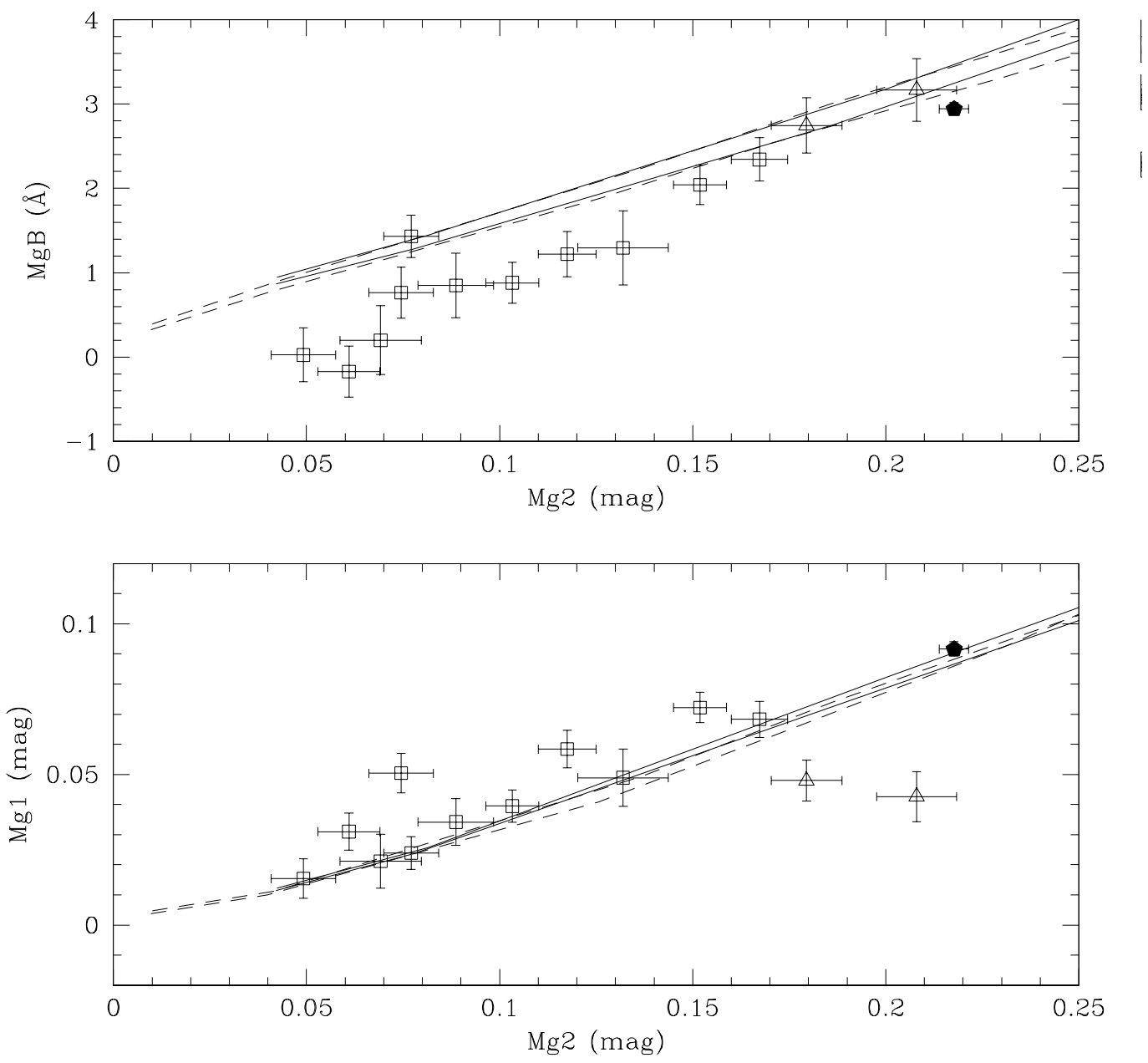

Fig. 7.- Figures comparing the Mg2 index with $\mathrm{Mg} b$ and $\mathrm{Mg}$. Thomas et al. 2003 models are plotted as lines. Solid lines represent a sequence of metallicity for a 14 Gyr old stellar population. The dashed lines are models for a 2 Gyr old SSP. For each age, the two individual lines represent two different $\alpha$-element ratios $([\alpha / \mathrm{Fe}]=0.0$ and 0.3$)$. Open squares are our 11 normal GCs, while open triangles represent the two GCs with emission. The filled pentagon represents the center of NGC 7457 itself. 


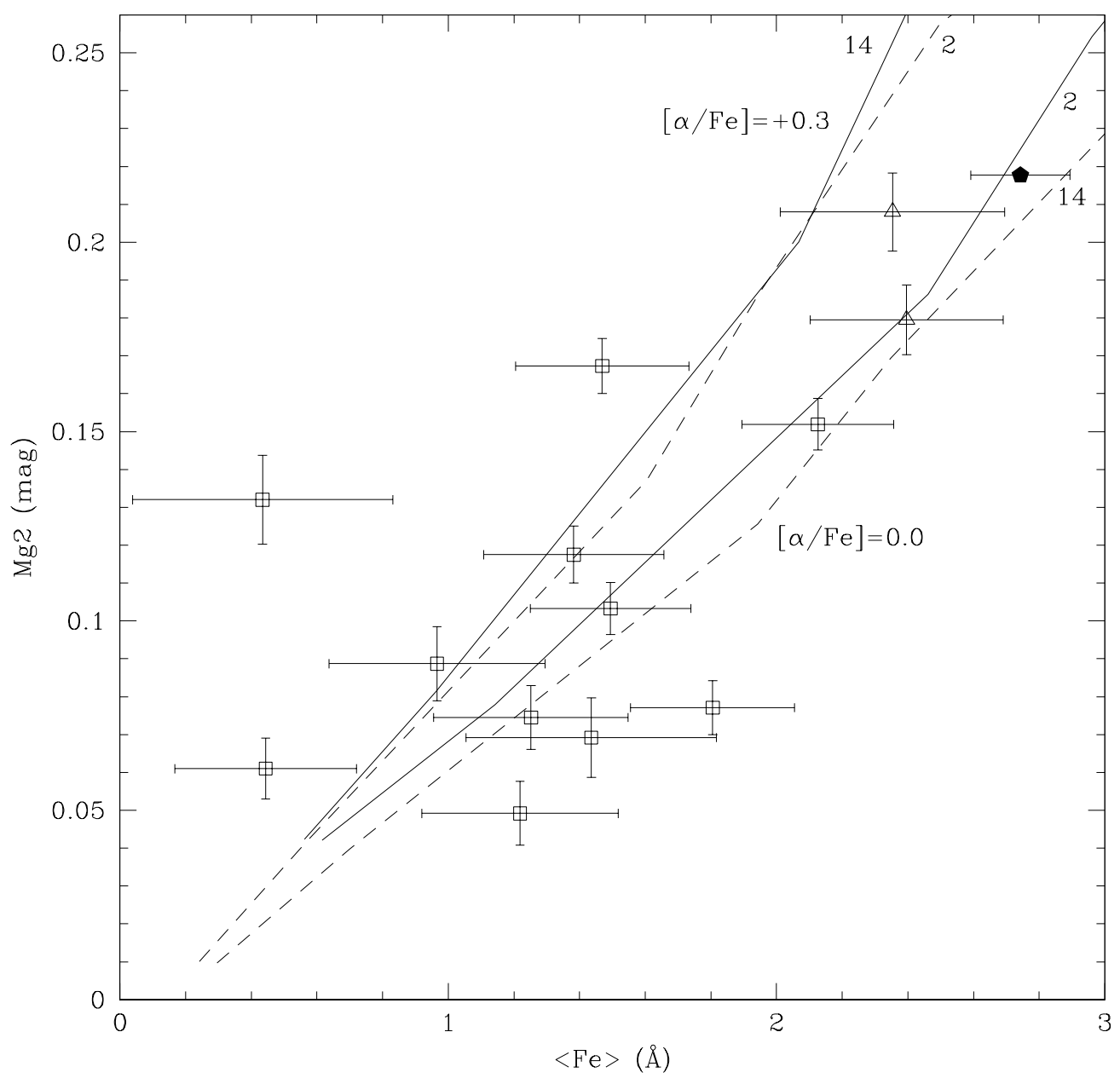

Fig. 8. - Plot of Mg2 vs. the $<\mathrm{Fe}>$ index. The symbols and model lines are as in Figure 7. Model lines are labeled with ages and $[\alpha / \mathrm{Fe}]$. 


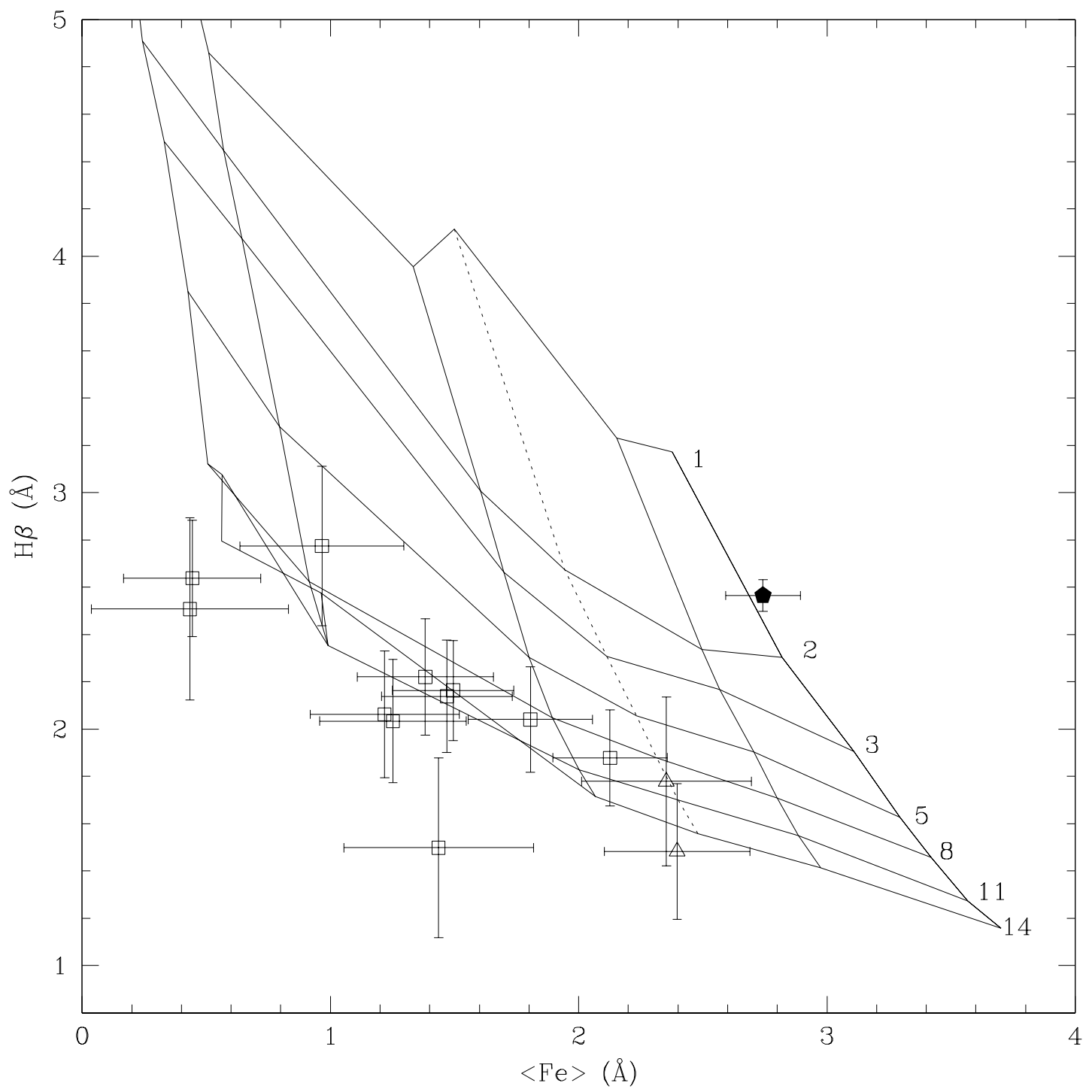

Fig. 9.- Plot of $\mathrm{H} \beta$ vs. $<\mathrm{Fe}>$ index, with a grid of model isochrone and isometallicity lines overlain (from Thomas et al. 2003, 2004). From left to right, the isometallicity lines represent $[Z / \mathrm{H}]$ $=-2.25,-1.35,-0.33,0.00,0.35,0.67$. The solar isometallicity line can be seen as the dotted line. All metallicities assume $[\alpha / \mathrm{Fe}]=0.3$. Ages are indicated at the right of each isochrone, in Gyr. Open squares are our 11 normal globular clusters, while open triangles represent the two GCs with emission. The filled pentagon represents the center of NGC 7457 itself. 


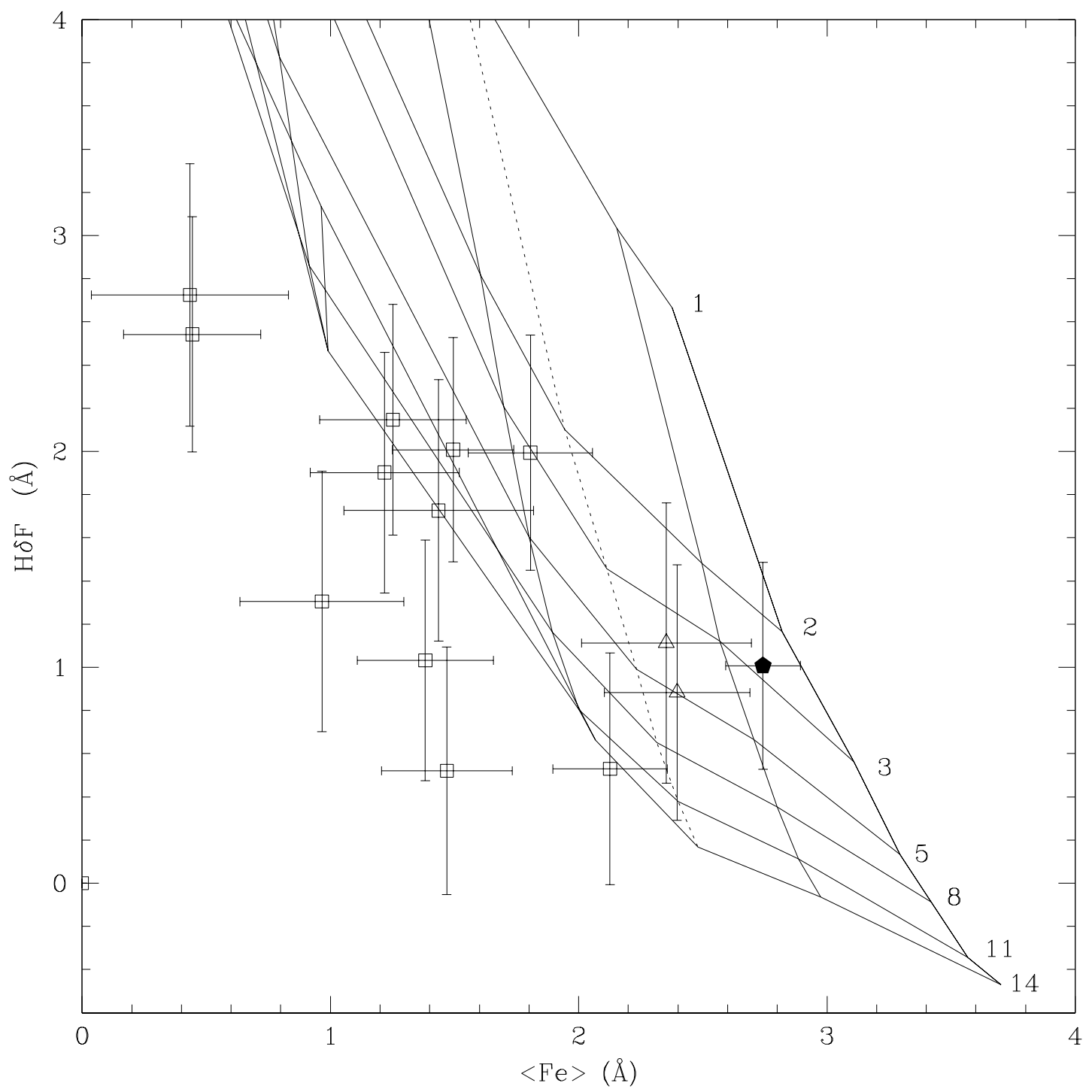

Fig. 10. - Plot of $\mathrm{H} \delta \mathrm{F}$ vs. $<\mathrm{Fe}>$ index. Symbols and grids as in Figure 9. 


\section{GC6}

\section{GC7}

Fig. 11. - Two-dimensional spectra for GC6 and GC7, showing the [O III] $\lambda \lambda 49599,5007$ emission lines. Shorter wavelengths are to the left. The [O III] lines in GC6 are significantly fainter than those in $\mathrm{GC} 7$, so the [O III] $\lambda 4959$ line is not visible here for this cluster. Notice the possible diffuse emission slightly redward of the compact [O III] $\lambda 5007$ emission.

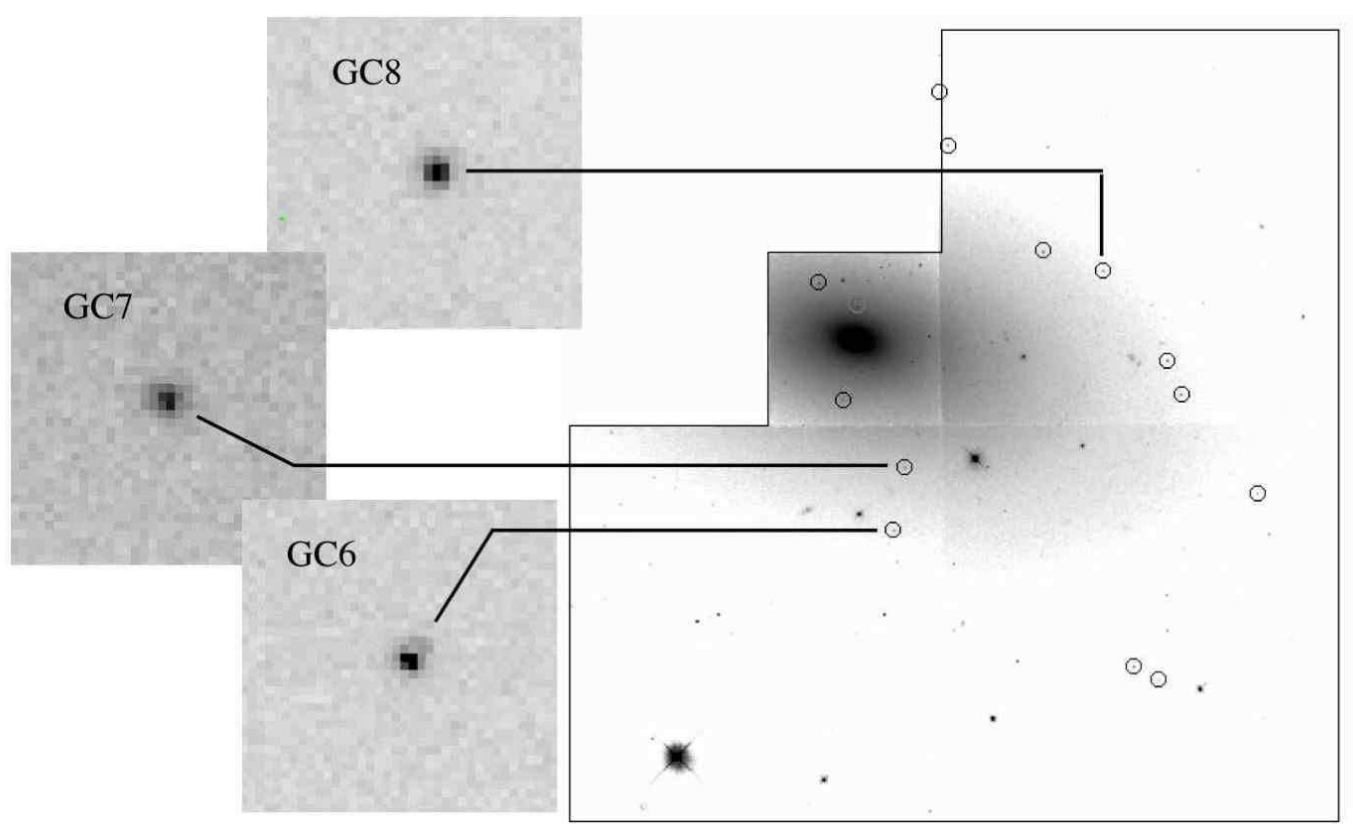

Fig. 12.- WFPC2 F814W image of NGC 7457, with our 13 spectroscopic GC targets marked by black circles. The two GCs which exhibit emission lines are shown in detail on the left, alongside GC8, a normal GC. 

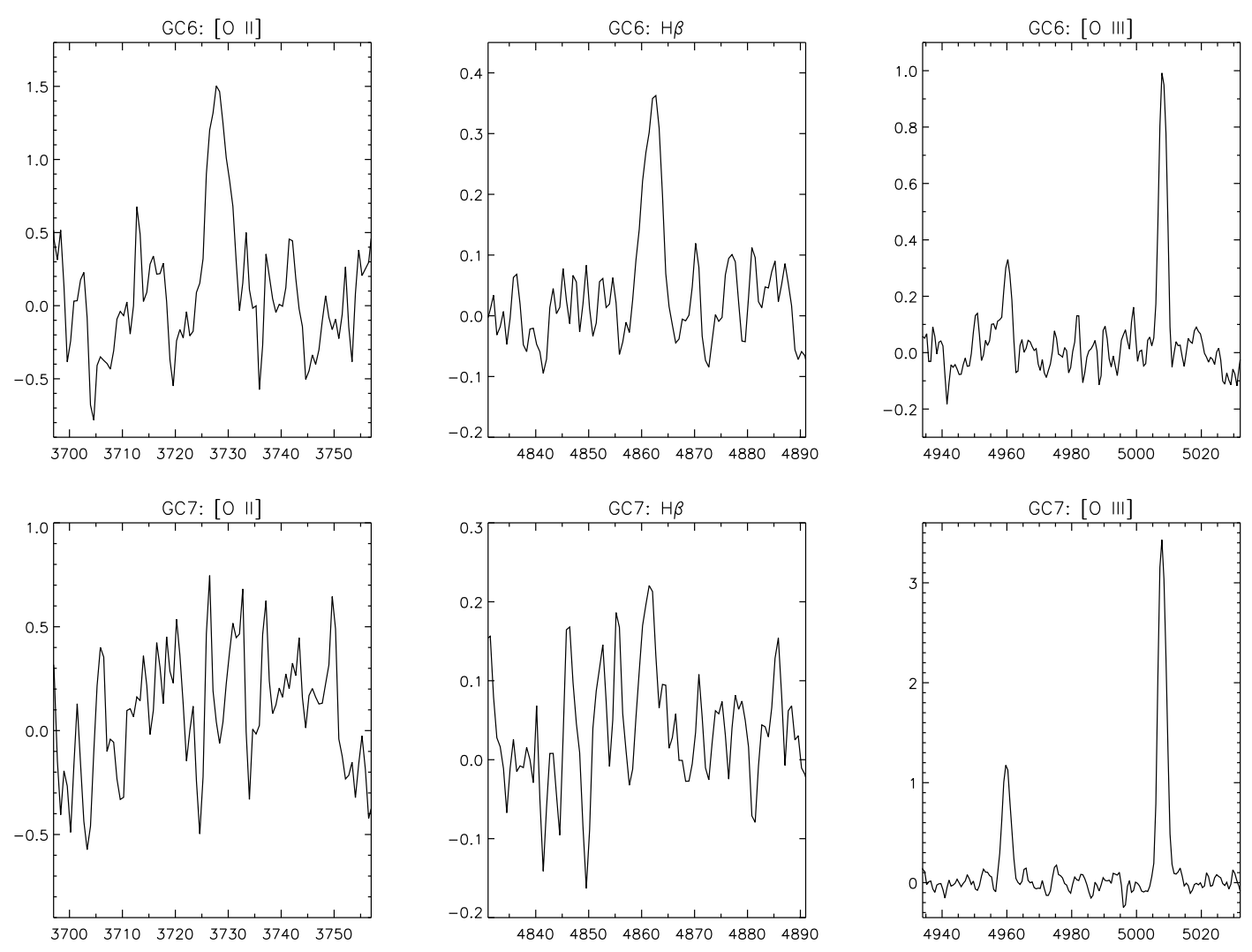

Fig. 13.- Detail of emission line spectra for GC6 and GC7. These are segments of the original spectrum, which have been normalized and had a 2.0 Gyr old, solar metallicity MILES SSP model subtracted from them. Clearly, $\mathrm{H} \beta$ and $[\mathrm{O}$ II] were too weak to be reliably measured in GC7. 


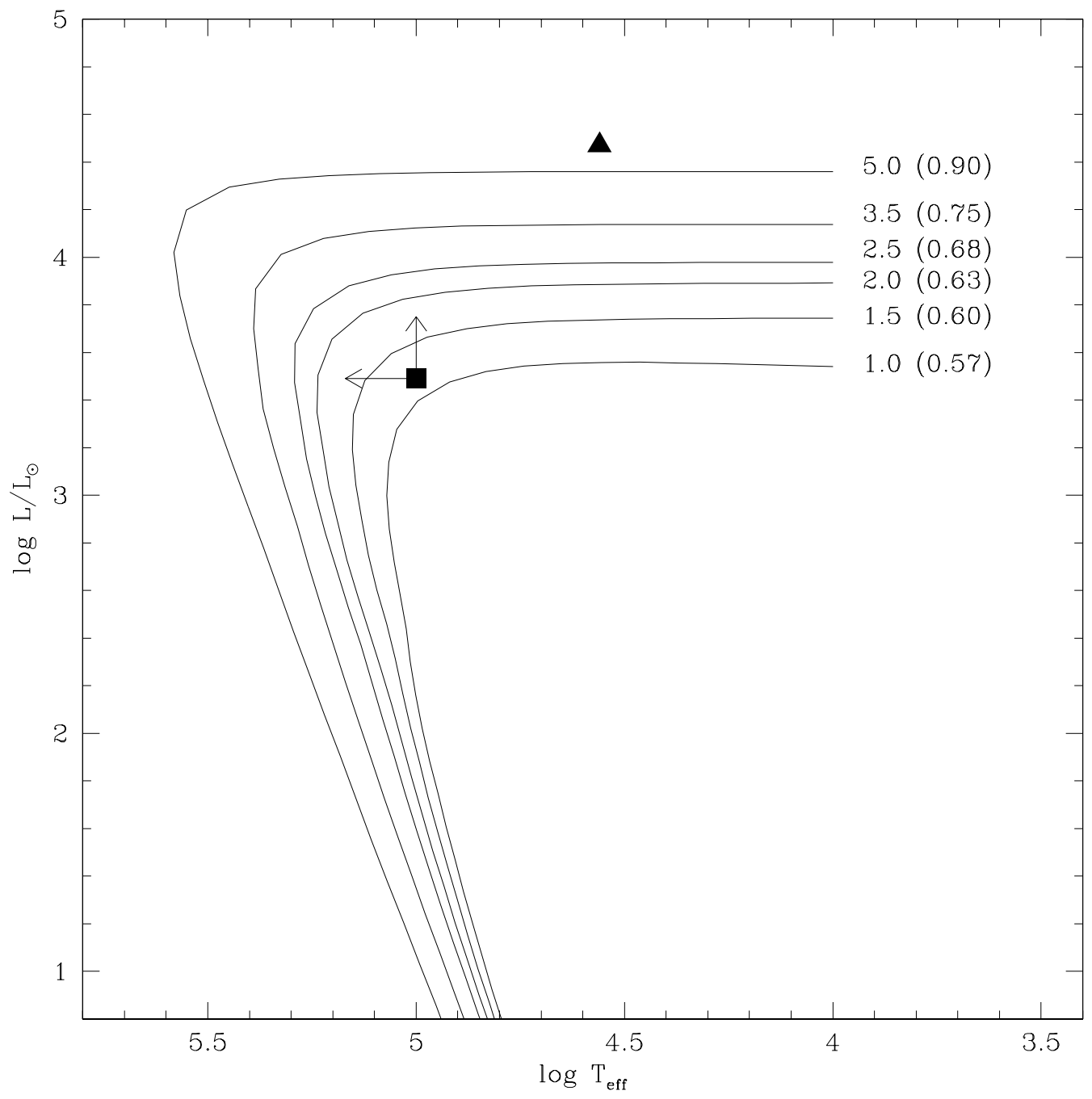

Fig. 14.- Model tracks for post-AGB stellar evolution from Vassiliadis \& Wood 1994, with GC6 overplotted as a triangle and GC7 represented by a square. Note that the plotted luminosity and temperature are both lower limits for GC7. The models are for metallicity $\mathrm{Z}=0.016$, and each track is labeled with the mass of the progenitor main-sequence star, and in parentheses, the core mass at $10^{4} \mathrm{~K}$. 
Table 1. Global Parameters for NGC 7457

\begin{tabular}{lcc}
\hline \hline Parameter & Value & Source \\
\hline Type & SA(rs)0-? & NED \\
R.A. (2000) (h:m:s) & $23: 00: 59.9$ & NED \\
Dec. (2000) (d:m:s) & $+30: 08: 42$ & NED \\
Radial Vel. (km s $\left.{ }^{-1}\right)$ & $812 \pm 6$ & NED \\
$\mathrm{m}_{-} \mathrm{M}$ & $30.55 \pm 0.21$ & Cappellari et al. 2006 \\
$\mathrm{M}_{V}$ & $-19.35 \pm 0.24$ & de Vaucouleurs et al. 1991 \\
$(\mathrm{B}-\mathrm{V})_{0}$ & 0.81 & HyperLeda \\
$\mathrm{A}_{V}$ & 0.17 & Schlegel et al. 1998 \\
Inclination $($ degrees $)$ & 76 & HyperLeda \\
Dust? & None & Peletier et al. 1999 \\
$\mathrm{H}_{2}$ Mass $\left(\mathrm{M}_{\odot}\right)$ & $(3.3 \pm 1.0) \times 10^{6}$ & Welch \& Sage 2003 \\
H I Mass $\left(\mathrm{M}_{\odot}\right)$ & $(1.6 \pm 0.2) \times 10^{6}$ & Sage \& Welch 2006 \\
\hline
\end{tabular}

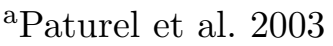

Table 2. UV Magnitudes and Colors

\begin{tabular}{|c|c|c|c|c|}
\hline $\begin{array}{l}\text { R.A. (2000) } \\
\text { (hr:min:sec) }\end{array}$ & $\begin{array}{c}\text { Dec. }(2000) \\
\left({ }^{\circ}:^{\prime \prime}{ }^{\prime \prime}\right)\end{array}$ & $\begin{array}{c}\text { F330W } \\
\text { (mag) }\end{array}$ & $\begin{array}{l}\text { V-F330W } \\
\text { (mag) }\end{array}$ & ID \\
\hline 23:01:01.02 & $+30: 08: 43.5$ & $22.49 \pm 0.03$ & $0.63 \pm 0.04$ & GC11 \\
\hline 23:00:59.31 & $+30: 08: 53.5$ & $22.53 \pm 0.03$ & $0.67 \pm 0.04$ & UV1 \\
\hline 23:01:00.82 & $+30: 08: 47.5$ & $21.17 \pm 0.02$ & $0.69 \pm 0.03$ & L1 \\
\hline 23:00:59.56 & $+30: 08: 38.6$ & $23.52 \pm 0.05$ & $0.75 \pm 0.07$ & UV2 \\
\hline 23:01:00.35 & $+30: 08: 44.6$ & $23.00 \pm 0.04$ & $0.96 \pm 0.05$ & UV3 \\
\hline 23:00:59.19 & $+30: 08: 47.5$ & $22.55 \pm 0.03$ & $0.98 \pm 0.04$ & UV4 \\
\hline 23:01:00.36 & $+30: 08: 46.4$ & $22.59 \pm 0.03$ & $1.02 \pm 0.04$ & UV5 \\
\hline 23:01:00.24 & $+30: 09: 01.2$ & $23.35 \pm 0.04$ & $1.20 \pm 0.05$ & UV6 \\
\hline 23:00:59.41 & $+30: 08: 32.3$ & $22.51 \pm 0.03$ & $1.25 \pm 0.04$ & GC10 \\
\hline
\end{tabular}


Table 3. Basic Data for Spectroscopic Globular Clusters in NGC 7457

\begin{tabular}{|c|c|c|c|c|c|c|c|c|c|}
\hline ID & $\begin{array}{l}\text { R.A. }(2000) \\
\text { (hr:min:sec) }\end{array}$ & $\begin{array}{c}\text { Dec. }(2000) \\
\left({ }^{\circ}:^{\prime \prime} !^{\prime \prime}\right)\end{array}$ & $\begin{array}{c}V \\
(\mathrm{mag})\end{array}$ & $\begin{array}{l}V-I \\
(\mathrm{mag})\end{array}$ & $\begin{array}{c}\mathrm{RV}^{\mathrm{a}} \\
\left(\mathrm{km} \mathrm{s}^{-1}\right)\end{array}$ & $\begin{array}{l}\mathrm{GC} \mathrm{R}_{e f f} \mathrm{~b} \\
\quad(\mathrm{pc})\end{array}$ & $\begin{array}{l}\text { Proj. Rad. } \\
\text { (") }\end{array}$ & $\begin{array}{c}{[\mathrm{m} / \mathrm{H}]} \\
(\mathrm{dex})\end{array}$ & $\mathrm{S} / \mathrm{N}^{\mathrm{c}}$ \\
\hline GC1 & 23:00:53.0 & 30:08:44.4 & $22.78 \pm 0.03$ & $1.03 \pm 0.05$ & $860 \pm 15$ & 3.2 & 105 & $-1.17 \pm 0.18$ & 21 \\
\hline GC2 & 23:00:53.4 & $30: 08: 42.3$ & $22.49 \pm 0.03$ & $0.93 \pm 0.04$ & $736 \pm 27$ & 4.0 & 99 & $-1.30 \pm 0.15$ & 26 \\
\hline GC3 & 23:00:54.2 & 30:09:23.3 & $22.30 \pm 0.02$ & $0.98 \pm 0.03$ & $908 \pm 20$ & 1.8 & 96 & $-1.11 \pm 0.13$ & 32 \\
\hline GC4 & $23: 00: 56.1$ & $30: 09: 24.2$ & $22.47 \pm 0.03$ & $0.96 \pm 0.04$ & $944 \pm 30$ & 1.7 & 72 & $-1.33 \pm 0.17$ & 18 \\
\hline GC5 & $23: 00: 56.7$ & 30:09:26.4 & $21.97 \pm 0.02$ & $1.15 \pm 0.03$ & $918 \pm 18$ & 4.1 & 67 & $-0.59 \pm 0.06$ & 35 \\
\hline GC6 & $23: 00: 57.4$ & 30:08:23.3 & $22.43 \pm 0.03$ & $1.20 \pm 0.04$ & $818 \pm 11$ & 0.8 & 44 & $-0.28 \pm 0.12$ & 25 \\
\hline GC7 & 23:00:58.0 & 30:08:33.1 & $22.34 \pm 0.02$ & $1.05 \pm 0.03$ & $822 \pm 10$ & 12.3 & 32 & $-0.35 \pm 0.18$ & 20 \\
\hline GC8 & 23:00:58.4 & 30:09:28.1 & $22.14 \pm 0.02$ & $1.11 \pm 0.03$ & $891 \pm 11$ & 3.4 & 52 & $-0.94 \pm 0.18$ & 29 \\
\hline GC9 & 23:00:59.2 & 30:09:21.5 & $21.76 \pm 0.02$ & $0.97 \pm 0.02$ & $941 \pm 32$ & 1.8 & 41 & $-1.56 \pm 0.07$ & 28 \\
\hline GC10 & 23:00:59.4 & $30: 08: 32.3$ & $21.26 \pm 0.01$ & $1.14 \pm 0.02$ & $817 \pm 9$ & 1.0 & 14 & $-0.67 \pm 0.10$ & 30 \\
\hline N7457 & 23:01:00.0 & $30: 08: 42.5$ & & & $789 \pm 15$ & & 2 & $-0.28 \pm 0.15$ & 583 \\
\hline GC11 & 23:01:01.0 & $30: 08: 43.5$ & $21.86 \pm 0.02$ & $1.00 \pm 0.03$ & $721 \pm 19$ & 2.1 & 15 & $-0.93 \pm 0.06$ & 35 \\
\hline GC12 & 23:01:01.4 & $30: 09: 20.6$ & $21.99 \pm 0.02$ & $1.01 \pm 0.03$ & $919 \pm 33$ & 2.5 & 43 & $-1.11 \pm 0.12$ & 27 \\
\hline GC13 & $23: 01: 02.1$ & $30: 09: 26.3$ & $22.84 \pm 0.03$ & $0.89 \pm 0.05$ & $834 \pm 27$ & 3.3 & 53 & $-1.27 \pm 0.09$ & 18 \\
\hline
\end{tabular}

${ }^{a}$ All radial velocities are heliocentric.

${ }^{\mathrm{b}}$ Assuming a distance of $13 \mathrm{Mpc}$

${ }^{\mathrm{c}}$ Average signal-to-noise ratio per resolution element over the bandpass of the $\mathrm{H} \beta$ Lick/IDS index 
Table 4. Lick/IDS Indices

\begin{tabular}{|c|c|c|c|c|c|c|c|c|c|c|}
\hline ID & $\begin{array}{l}\mathrm{H} \beta \\
(\AA)\end{array}$ & $\begin{array}{c}\mathrm{H} \gamma_{F} \\
(\AA)\end{array}$ & $\begin{array}{r}\mathrm{H} \delta_{F} \\
(\AA)\end{array}$ & $\begin{array}{c}\mathrm{CN}_{2} \\
(\mathrm{mag})\end{array}$ & $\begin{array}{c}\mathrm{Ca} 4227 \\
(\AA)\end{array}$ & $\begin{array}{c}\mathrm{Fe} 4383 \\
(\AA)\end{array}$ & $\begin{array}{c}\text { Fe5270 } \\
(\AA)\end{array}$ & $\begin{array}{c}\text { Fe5335 } \\
(\AA)\end{array}$ & $\begin{array}{l}\mathrm{Mg} b \\
(\AA)\end{array}$ & $\begin{array}{l}\text { Mg2 } \\
\text { (mag) }\end{array}$ \\
\hline GC1 & $2.77 \pm 0.34$ & $0.63 \pm 0.34$ & $1.30 \pm 0.60$ & $-0.05 \pm 0.02$ & $1.43 \pm 0.33$ & $2.56 \pm 0.75$ & $0.95 \pm 0.40$ & $0.98 \pm 0.52$ & $0.85 \pm 0.38$ & $0.09 \pm 0.010$ \\
\hline GC2 & $2.06 \pm 0.27$ & $1.67 \pm 0.28$ & $1.90 \pm 0.56$ & $0.01 \pm 0.02$ & $0.75 \pm 0.29$ & $-0.37 \pm 0.62$ & $1.49 \pm 0.38$ & $0.96 \pm 0.46$ & $0.03 \pm 0.32$ & $0.05 \pm 0.008$ \\
\hline GC3 & $2.04 \pm 0.22$ & $0.89 \pm 0.26$ & $1.99 \pm 0.54$ & $-0.05 \pm 0.02$ & $0.59 \pm 0.28$ & $0.78 \pm 0.52$ & $1.91 \pm 0.31$ & $1.70 \pm 0.39$ & $1.43 \pm 0.25$ & $0.08 \pm 0.007$ \\
\hline GC4 & $2.51 \pm 0.39$ & $0.79 \pm 0.39$ & $2.72 \pm 0.61$ & $-0.04 \pm 0.03$ & $0.63 \pm 0.39$ & $0.21 \pm 0.90$ & $1.49 \pm 0.50$ & $-0.62 \pm 0.61$ & $1.30 \pm 0.44$ & $0.13 \pm 0.012$ \\
\hline GC5 & $1.88 \pm 0.20$ & $0.06 \pm 0.24$ & $0.53 \pm 0.54$ & $0.05 \pm 0.02$ & $1.04 \pm 0.27$ & $2.59 \pm 0.46$ & $2.03 \pm 0.29$ & $2.22 \pm 0.36$ & $2.04 \pm 0.23$ & $0.15 \pm 0.007$ \\
\hline GC6 & $1.48 \pm 0.29$ & $-0.90 \pm 0.32$ & $0.88 \pm 0.59$ & $0.04 \pm 0.02$ & $1.62 \pm 0.31$ & $4.22 \pm 0.62$ & $2.69 \pm 0.37$ & $2.10 \pm 0.46$ & $2.75 \pm 0.33$ & $0.18 \pm 0.009$ \\
\hline GC7 & $1.78 \pm 0.36$ & $-1.25 \pm 0.38$ & $1.11 \pm 0.65$ & $-0.02 \pm 0.02$ & $1.60 \pm 0.36$ & $8.07 \pm 0.70$ & $2.19 \pm 0.44$ & $2.52 \pm 0.52$ & $3.17 \pm 0.37$ & $0.21 \pm 0.010$ \\
\hline GC8 & $2.22 \pm 0.25$ & $1.22 \pm 0.27$ & $1.03 \pm 0.56$ & $-0.07 \pm 0.02$ & $1.27 \pm 0.29$ & $3.05 \pm 0.54$ & $1.43 \pm 0.35$ & $1.34 \pm 0.43$ & $1.22 \pm 0.27$ & $0.12 \pm 0.008$ \\
\hline GC9 & $2.64 \pm 0.25$ & $1.46 \pm 0.26$ & $2.54 \pm 0.54$ & $-0.04 \pm 0.02$ & $0.28 \pm 0.29$ & $0.59 \pm 0.53$ & $0.60 \pm 0.34$ & $0.28 \pm 0.44$ & $-0.17 \pm 0.30$ & $0.06 \pm 0.008$ \\
\hline GC10 & $2.14 \pm 0.24$ & $-0.14 \pm 0.28$ & $0.52 \pm 0.57$ & $0.03 \pm 0.02$ & $0.86 \pm 0.30$ & $3.20 \pm 0.54$ & $1.51 \pm 0.34$ & $1.43 \pm 0.40$ & $2.34 \pm 0.26$ & $0.17 \pm 0.007$ \\
\hline N7457 & $2.56 \pm 0.07$ & $-0.04 \pm 0.13$ & $1.01 \pm 0.48$ & $0.04 \pm 0.01$ & $1.22 \pm 0.21$ & $4.69 \pm 0.10$ & $2.85 \pm 0.16$ & $2.63 \pm 0.25$ & $2.94 \pm 0.07$ & $0.22 \pm 0.004$ \\
\hline GC11 & $2.16 \pm 0.21$ & $1.28 \pm 0.22$ & $2.01 \pm 0.52$ & $-0.06 \pm 0.02$ & $0.63 \pm 0.26$ & $1.26 \pm 0.43$ & $1.68 \pm 0.30$ & $1.31 \pm 0.38$ & $0.88 \pm 0.24$ & $0.10 \pm 0.007$ \\
\hline GC12 & $2.03 \pm 0.26$ & $1.42 \pm 0.26$ & $2.15 \pm 0.54$ & $-0.02 \pm 0.02$ & $0.38 \pm 0.29$ & $1.18 \pm 0.58$ & $1.23 \pm 0.37$ & $1.28 \pm 0.46$ & $0.77 \pm 0.30$ & $0.07 \pm 0.0080$ \\
\hline GC13 & $1.50 \pm 0.38$ & $0.59 \pm 0.36$ & $1.73 \pm 0.61$ & $-0.02 \pm 0.02$ & $0.95 \pm 0.36$ & $1.10 \pm 0.79$ & $1.18 \pm 0.50$ & $1.70 \pm 0.57$ & $0.20 \pm 0.41$ & $0.07 \pm 0.011^{\mid}$ \\
\hline
\end{tabular}


Table 5. Multi-Line Fits

\begin{tabular}{lcccccc}
\hline \hline ID & $\begin{array}{c}\text { Age } \\
(\mathrm{Gyr})\end{array}$ & {$[\mathrm{Fe} / \mathrm{H}]$} & {$[\alpha / \mathrm{Fe}]$} & {$[\mathrm{Z} / \mathrm{H}]$} & $\chi^{2}$ & $\#$ indices \\
& $11 \pm 5$ & $-1.3 \pm 0.3$ & $0.4 \pm 0.3$ & $-1.0 \pm 0.2$ & 19.8 & 15 \\
GC1 & $13 \pm 4$ & $-1.6 \pm 0.3$ & $0.4 \pm 0.4$ & $-1.2 \pm 0.1$ & 17.6 & 13 \\
GC2 & $12 \pm 2$ & $-1.4 \pm 0.2$ & $0.3 \pm 0.2$ & $-1.2 \pm 0.1$ & 11.9 & 15 \\
GC3 & $12 \pm 4$ & $-1.6 \pm 0.3$ & $0.5 \pm 0.3$ & $-1.2 \pm 0.2$ & 10.0 & 15 \\
GC4 & $11 \pm 0.2$ & $-0.6 \pm 0.2$ & 5.1 & 13 \\
GC5 & $12 \pm 3$ & $-0.9 \pm 0.2$ & $0.3 \pm 0.2$ & $-0.6 \pm 0.1$ & 5.6 & 14 \\
GC6 & $11 \pm 3$ & $-0.5 \pm 0.1$ & $0.4 \pm 0.1$ & $-0.1 \pm 0.1$ & 9.2 & 12 \\
GC6 & $15 \pm 3$ & $-0.4 \pm 0.1$ & $0.3 \pm 0.1$ & $-0.1 \pm 0.1$ & 3.2 \\
GC7 & $15 \pm 6$ & $-0.3 \pm 0.2$ & $0.1 \pm 0.2$ & $-0.2 \pm 0.1$ & 18.4 & 8 \\
GC7 & $11 \pm 6$ & $-0.4 \pm 0.3$ & $0.2 \pm 0.2$ & $-0.2 \pm 0.2$ & 13.6 & 13 \\
GC8 & $7 \pm 3$ & $-0.8 \pm 0.2$ & $0.3 \pm 0.2$ & $-0.5 \pm 0.2$ & 8.0 & 14 \\
GC9 & $9 \pm 3$ & $-1.8 \pm 0.2$ & $0.7 \pm 0.2$ & $-1.12 \pm 0.2$ & 4.9 & 15 \\
GC10 & $11 \pm 4$ & $-0.8 \pm 0.2$ & $0.2 \pm 0.2$ & $-0.7 \pm 0.1$ & 7.9 & 17 \\
N7457 & $2.5 \pm 0.3$ & $0.18 \pm 0.06$ & $-0.03 \pm 0.03$ & $0.15 \pm 0.06$ & 7.2 & 15 \\
GC11 & $13 \pm 3$ & $-1.5 \pm 0.2$ & $0.3 \pm 0.3$ & $-1.2 \pm 0.1$ & 8.2 & 11 \\
GC12 & $12 \pm 3$ & $-1.5 \pm 0.3$ & $0.3 \pm 0.3$ & $-1.2 \pm 0.1$ & 5.0 & 15 \\
GC13 & $12 \pm 3$ & $-1.5 \pm 0.3$ & $0.3 \pm 0.3$ & $-1.2 \pm 0.2$ & 17.7 & \\
\hline
\end{tabular}

**All Balmer lines were excluded from these fits, due to the possibility that they are filled in by emission.

Table 6. Observed Emission Lines

\begin{tabular}{lcc}
\hline \hline Line & GC6 & GC7 \\
\hline$[\mathrm{O}$ II $] \lambda 3727$ & Strong & None \\
{$[$ Ne III $] \lambda 3869$} & None & Weak \\
{$[$ Ne III $] \lambda 3967$} & None & Weak \\
H $\beta$ & Moderate & Very Weak \\
{$[\mathrm{O}$ III $] \lambda 4959$} & Strong & Very Strong \\
{$[\mathrm{O}$ III $] \lambda 5007$} & Strong & Very Strong \\
\hline
\end{tabular}

Table 7. Emission Line Ratios for GC6

\begin{tabular}{lcc}
\hline \hline Ratio & MILES 2.0 Gyr & MILES 12.6 Gyr \\
\hline$[\mathrm{O}$ II $] \lambda 3727 / \mathrm{H} \beta$ & $2.9 \pm 0.4$ & $4.3 \pm 0.9$ \\
{$[\mathrm{O}$ III $] \lambda 5007 / \mathrm{H} \beta$} & $2.3 \pm 0.3$ & $3.9 \pm 0.7$ \\
{$[\mathrm{O}$ II $] \lambda 3727 /[\mathrm{O}$ III $] \lambda 5007$} & $1.3 \pm 0.1$ & $1.1 \pm 0.1$ \\
\hline
\end{tabular}

